\title{
A CERTAIN PROPERTY OF GEODESICS OF THE FAMILY OF RIEMANNIAN MANIFOLDS $O_{n}^{2}$ (IV)
}

\author{
By Tominosuke OTSUKI
}

\section{$\S 0$. Introduction.}

This is exactly a continuation of Part (III) ([13]) with the same title written by the present author. We shall use the same notation in it.

The period $T$ of any non-constant solution of $x(t)$ of the non-linear differential equation of order 2 :

$$
n x\left(1-x^{2}\right) \frac{d^{2} x}{d t^{2}}+\left(\frac{d x}{d t}\right)^{2}+\left(1-x^{2}\right)\left(n x^{2}-1\right)=0
$$

with a constant $n>1$ such that $x^{2}+x^{\prime 2}<1$ is given by the integral:

$$
T=\sqrt{n c} \int_{x_{0}}^{x_{1}} \frac{d x}{x \sqrt{(n-x)\left\{x(n-x)^{n-1}-c\right\}}},
$$

where $0<x_{0}<1<x_{1}<n$ and $c=x_{0}\left(n-x_{0}\right)^{n-1}=x_{1}\left(n-x_{1}\right)^{n-1}$.

We shall show in the present work that the following conjecture is true for $n \geqq 84$.

CONJECTURe C. The period function $T$ as a function of $\tau=\left(x_{1}-1\right) /(n-1)$ and $n$ is monotone decreasing with respect to $n(\geqq 2)$ for any fixed $\tau(0<\tau<1)$.

\section{$\S 1$. Fundamental formulas.}

Denoting $T$ by $\Omega(\tau, n)$, we have by (7.4) and Proposition 3 in [13] the following formula:

$$
\frac{\partial \Omega(\tau, n)}{\partial n}=-\frac{\sqrt{c / n}}{2 b^{2} n} \int_{x_{0}}^{1} \frac{(1-x) \sqrt{x(n-x)^{n-1}-c}}{x^{2}(n-x)^{n}} V\left(x, x_{1}\right) d x,
$$

where $b=\sqrt{B-c}, B=(n-1)^{n-1}$ and $V\left(x, x_{1}\right)$ is defined as follows: By (2.10), (4.2), (1.4), (1.5), (7.1), (7.7) and (7.10) in [13], respectively,

$$
\begin{gathered}
f_{0}(z):=(2 n-1-z) B-(n-z)^{n-1}\left\{n-z+(n-1) z^{2}\right\}, \\
F_{2}(z):=-\left\{(2 n+1) z^{2}-2\left(2 n^{2}+5 n-4\right) z+16 n^{2}-16 n+3\right\} B \\
+(n-z)^{n-1}\left\{-(n-1) z^{3}+\left(2 n^{2}-7 n+8\right) z^{2}+(n-3)(4 n-1) z+3 n(2 n-1)\right\},
\end{gathered}
$$

Received November 14, 1980. 


$$
\begin{gathered}
\lambda(z):=\log (n-z)+\frac{n-1}{n-z}, \quad \phi(z):=z(n-z)^{n-1}, \\
\tilde{\lambda}(z):=\lambda(z)-\frac{n}{n-1} \cdot \frac{(z-1)^{2}}{z(n-z)}=\log (n-z)+\frac{n z-1}{(n-1) z}, \\
N\left(z, x_{1}\right):=(n-z) F_{2}(z)\left\{\lambda(z)-\tilde{\lambda}\left(x_{1}\right)\right\}+3(z-1)^{2} f_{0}(z) \\
-2 n(z-1)^{3}\left\{B-z(n-z)^{n-1}\right\}, \\
V\left(x, x_{1}\right):=\frac{x^{2} N\left(x, x_{1}\right)}{(1-x)^{5} \sqrt{n-x}}+\frac{X^{2} N\left(X, x_{1}\right)}{(X-1)^{5} \sqrt{n-X}},
\end{gathered}
$$

where $X=X_{n}(x), 0<x<1<X<n$, defined by

$$
\phi(x)=\phi(X) .
$$

We know the following facts about $V\left(x, x_{1}\right)$ by Lemma 8.1 in [13].

(i) $\lim _{x_{1} \rightarrow n-0} V\left(x, x_{1}\right)=+\propto$ for $0<x<1$;

(ii) $V\left(x, x_{1}\right)$ is increasing with respect to $x_{1}$ for each $x(0<x<1)$;

(iii) When $n>2, \lim _{x \rightarrow+0} V(x, X(x))=0$ and furthermore when $n>(5+\sqrt{13}) / 4 \dot{\doteqdot}$ 2.15, $V(x, X(x))>0$ near $x=0$;

(iv) When $n>2, \lim _{x \rightarrow 1-0} V(x, X(x))=0$ and furthermore when $n>(1+\sqrt{ } 13) / 2 \doteqdot$ 2.30, $V(x, X(x))>0$ near $x=1$.

From these facts, we obtain $V\left(x, x_{1}\right)>0$ for $0<x<1, X(x)<x_{1}<n$ if we can prove

$$
V(x, X(x)) \geqq 0 \quad \text { for } 0<x<1 .
$$

By (8.2) and (8.3) in [13], setting for $0<x<n, x \neq 1$

$$
\begin{aligned}
U_{1}(x) & :=\frac{3 x^{2} f_{0}(x)}{(x-1)^{3} \sqrt{n-x}}, \\
U_{2}(x): & =\frac{2 n x^{2}\{B-\psi(x)\}}{(1-x)^{2} \sqrt{n-x}}, \\
U_{3}(x): & =\frac{n x F_{2}(x)}{(n-1)(x-1)^{3} \sqrt{n-x}}
\end{aligned}
$$

and for $0<x<1$

$$
\begin{gathered}
U_{0}(x):=\frac{x^{2} \sqrt{n-x}}{(1-x)^{5}} F_{2}(x)\{\lambda(x)-\tilde{\lambda}(X(x))\}, \\
U_{4}(x):=U_{3}(X(x)), \quad U_{5}(x):=U_{1}(X(x)), \quad U_{6}(x):=U_{2}(X(x)),
\end{gathered}
$$

we have from (8.1) in [13] the expression of $V(x, X(x))$ as follows: 


$$
V(x, X(x))=U_{0}(x)-U_{1}(x)+U_{2}(x)+U_{4}(x)+U_{5}(x)-U_{6}(x),
$$

in which $U_{i}(x), i=0,1,2,4,5,6$, are all positive for $0<x<1$.

\section{$\S 2$. Certain auxiliary inequalities (I).}

Proposition 1. When $n>2$, we have $n-(n-1) x^{1 /(n-1)}<X(x)$ for $0<x<1$.

Proof. Setting $Y=Y(x):=n-(n-1) x^{1 /(n-1)}$, we have

$$
Y(1)=1=X(1), \quad Y(0)=n=X(0) .
$$

Setting $x=1-t$ near $x=1$, we have

$$
\begin{aligned}
& Y(1-t)=n-(n-1)(1-t)^{1 /(n-1)} \\
& \quad=n-(n-1)\left\{1-\frac{t}{n-1}-\frac{(n-2) t^{2}}{2(n-1)^{2}}-\frac{(n-2)(2 n-3) t^{3}}{6(n-1)^{3}}-\cdots\right\} \\
& \quad=1+t+\frac{(n-2) t^{2}}{2(n-1)}+\frac{(n-2)(2 n-3) t^{3}}{6(n-1)^{2}}+\cdots
\end{aligned}
$$

and (8.12) in $[13]$

$$
X(1-t)=1+t+\frac{2(n-2) t^{2}}{3(n-1)}+\cdots,
$$

which implies $X(1-t)>Y(1-t)$ for sufficiently small $t$.

Now, we suppose that the above inequality is not true for the interval $0<$ $x<1$ and let $\xi$ be the maximum value of $x$ such that $X(x)=Y(x)$, i. e.

$$
X(\xi)=Y(\xi) \text { and } X(x)>Y(x) \quad \text { for } \xi<x<1 .
$$

Then, we must have

$$
0>\frac{d X}{d x} \geqq \frac{d Y}{d x} \quad \text { at } x=\xi \text {. }
$$

Since we have

$$
\frac{d X}{d x}=\frac{1-x}{x(n-x)} \cdot \frac{X(n-X)}{1-X}, \quad \frac{d Y}{d x}=-x^{1 /(n-1)-1},
$$

we obtain at $x=\xi$

$$
\begin{aligned}
\frac{d X}{d x} & =\frac{1-\xi}{\xi(n-\xi)} \cdot \frac{Y(\xi)(n-Y(\xi))}{1-Y(\xi)}=-\frac{(1-\xi) Y(\xi)}{\xi(n-\xi)} \cdot \frac{(n-1) \xi^{1 /(n-1)}}{(n-1)\left(1-\xi^{1 /(n-1)}\right)} \\
& =\frac{(1-\xi) Y(\xi)}{(n-\xi)\left(1-\xi^{1 /(n-1)}\right)} \frac{d Y}{d x} .
\end{aligned}
$$

Hence it must be 


$$
\frac{(1-\xi)\left\{n-(n-1) \xi^{1 /(n-1)}\right\}}{(n-\xi)\left(1-\xi^{1 /(n-1)}\right)} \leqq 1 .
$$

On the other hand, we shall show that

$$
\frac{(1-x)\left\{n-(n-1) x^{1 /(n-1)}\right\}}{(n-x)\left(1-x^{1 /(n-1)}\right)}>1 \quad \text { for } 0<x<1,
$$

which contradicts to (2.1). (2.2) is equivalent to

Since we have

$$
(n-1) x^{(n-2) /(n-1)}<1+(n-2) x \quad \text { for } 0<x<1 .
$$

$\left((n-1) x^{(n-2) /(n-1)}\right)^{\prime}=(n-2) x^{-1 /(n-1)}>0,\left((n-1) x^{(n-2) /(n-1)}\right)^{\prime \prime}=-\frac{n-2}{n-1} x^{-n /(n-1)}<0$,

the above inequality is clear.

Q.E.D.

Proposition 1 implies immediately the following

LEMMA 2.1. When $n>2$, we have

$$
x=X_{n}^{-1}(X)>\left(\frac{n-X}{n-1}\right)^{n-1} \quad \text { for } 1<X<n .
$$

LEMMA 2.2. When $n>2$, we have

$$
\frac{x^{2}}{(1-x)^{2} \sqrt{n-x}}>\frac{\sqrt{B(n-X)^{2(n-1)}}}{\left\{B-(n-X)^{n-1}\right\}^{2} \sqrt{n B-(n-X)^{n-1}}} \quad \text { for } 0<x<1 .
$$

Proof. Since we have for $0<x<n, x \neq 1$

$$
\frac{d}{d x} \frac{x^{2}}{(x-1)^{2} \sqrt{n-x}}=\frac{x\left(x^{2}+3 x-4 n\right)}{2(x-1)^{3}(n-x)^{3 / 2}}
$$

and $x^{2}+3 x-4 n<0$ for $0<x<1$, the function $x^{2} /(x-1)^{2} \sqrt{n-x}$ is increasing in the interval $0<x<1$. Therefore, by Lemma 2.1 we obtain

$$
\begin{aligned}
& \frac{x^{2}}{(1-x)^{2} \sqrt{n-x}}>\left[\frac{t^{2}}{(1-t)^{2} \sqrt{n-t}}\right]_{t=(n-X / n-1)^{n-1}} \\
& =\frac{(n-X)^{2 n-2}}{B^{2}} /\left\{1-\frac{(n-X)^{n-1}}{B}\right\}^{2} \sqrt{n-\frac{(n-X)^{n-1}}{B}} \\
& =\frac{\sqrt{B(n-X)^{2(n-1)}}}{\left\{B-(n-X)^{n}\right\}^{2} \sqrt{n B-(n-X)^{n-1}}} .
\end{aligned}
$$

LEMMA 2.3. When $n>2$, we have

$$
\frac{X^{2}}{(X-1)^{2} \sqrt{n-X}}-\frac{x^{2}}{(1-x)^{2} \sqrt{n-x}}<\frac{X^{2}\{1-\nu(X)\}}{(X-1)^{2} \sqrt{n-X}} \quad \text { for } 0<x<1 \text {, }
$$


where

$$
\nu(X):=\frac{\sqrt{B}(X-1)^{2}(n-X)^{2 n-3 / 2}}{X^{2}\left\{B-(n-X)^{n-1}\right\}^{2} \sqrt{n B-(n-X)^{n-1}}} \quad \text { for } 1<X<n
$$

Proof. From Lemma 2.2 we obtain immediately

$$
\begin{aligned}
& \frac{X^{2}}{(X-1)^{2} \sqrt{n-X}}-\frac{x^{2}}{(1-x)^{2} \sqrt{n-x}} \\
& <\frac{X^{2}}{(X-1)^{2} \sqrt{n-X}}-\frac{\sqrt{B(} n-X)^{2(n-1)}}{\left\{B-(n-X)^{n-1}\right\}^{2} \sqrt{n B-(n-X)^{n-1}}} \\
& =\frac{X^{2}}{(X-1)^{2} \sqrt{n-X}}\left\{1-\frac{\sqrt{B}(n-X)^{2 n-3 / 2}}{X^{2}\left\{B-(n-X)^{n-1}\right\}^{2} \sqrt{n B-(n-X)^{n-1}}}\right\} \text {. Q.E.D. }
\end{aligned}
$$

LEMMA 2.4. When $n>2$, we have

$$
U_{5}(x)-U_{1}(x)>\frac{3 n X f_{0}(X)}{(n-1)(X-1)^{2} \sqrt{n-X}} \quad \text { for } 0<x<1 .
$$

Proof. From (1.9) and (1.13) we obtain

$$
\begin{aligned}
& U_{5}(x)-U_{1}(x)=\frac{3 X^{2} f_{0}(X)}{(X-1)^{3} \sqrt{n-X}}-\frac{3 x^{2} f_{0}(x)}{(x-1)^{3} \sqrt{n-x}} \\
& =\frac{3 X^{2} f_{0}(X)}{(X-1)^{3} \sqrt{n-X}}-\frac{3 x}{n-x} \cdot \frac{x \sqrt{n-x} f_{0}(x)}{(x-1)^{3}}
\end{aligned}
$$

and by means of Proposition 2 in [12]

$$
\begin{aligned}
& <\frac{3 X^{2} f_{0}(X)}{(X-1)^{3} \sqrt{n-X}}-\frac{3 x}{n-x} \cdot \frac{X \sqrt{n-X} f_{0}(X)}{(X-1)^{3}} \\
= & \frac{3 X \sqrt{n-X} f_{0}(X)}{(X-1)^{3}} \cdot\left(\frac{X}{n-X}-\frac{x}{n-x}\right)=\frac{3 n X \sqrt{n-X} f_{0}(X)}{(X-1)^{3}} \cdot\left(\frac{1}{n-X}-\frac{1}{n-x}\right) \\
> & \frac{3 n X \sqrt{n-X} f_{0}(X)}{(X-1)^{3}}\left(\frac{1}{n-X}-\frac{1}{n-1}\right)=\frac{3 n X f_{0}(X)}{(n-1)(X-1)^{2} \sqrt{n-X}} \quad \text { for } 0<x<1 .
\end{aligned}
$$

Q.E.D.

LEMMA 2.5. When $n>2$, we have

$$
\begin{aligned}
& -U_{1}(x)+U_{2}(x)+U_{4}(x)+U_{5}(x)-U_{6}(x) \\
& >\frac{n X}{(n-1)(X-1)^{3} \sqrt{n-X}} \cdot\left\{G_{2}(X)+3(X-1) f_{0}(X)\right\} \quad \text { for } 0<x<1,
\end{aligned}
$$

where 


$$
G_{2}(x):=F_{2}(x)-2(n-1) x(x-1)\{B-\psi(x)\}\{1-\nu(x)\},
$$

and when $n>\left(13+\sqrt{153)} / 2 \doteqdot 12.685, G_{2}(x)+3(x-1) f_{0}(x)\right.$ is positive for $x>1$ sufficiently near $x=1$.

Proof. From (1.9), (1.10), (1.11) and (1.13) and by Lemma 2.3 and Lemma 2.4, we obtain

$$
\begin{aligned}
& -U_{1}(x)+U_{2}(x)+U_{4}(x)+U_{5}(x)-U_{6}(x) \\
= & U_{4}(x)-\left\{U_{6}(x)-U_{2}(x)\right\}+\left\{U_{5}(x)-U_{1}(x)\right\} \\
= & \frac{n X F_{2}(X)}{(n-1)(X-1)^{3} \sqrt{n-X}}-2 n\{B-\psi(X)\}\left\{\frac{X^{2}}{(X-1)^{2} \sqrt{n-X}}-\frac{x^{2}}{(1-x)^{2} \sqrt{n-x}}\right\} \\
& +\left\{U_{5}(x)-U_{1}(x)\right\}>\frac{n X F_{2}(X)}{(n-1)(X-1)^{3} \sqrt{n-X}}-2 n\{B-\phi(X)\} \frac{X^{2}\{1-\nu(X)\}}{(X-1)^{2} \sqrt{n-X}} \\
& +\frac{3 n X f_{0}(X)}{(n-1)(X-1)^{2} \sqrt{n-X}}=\frac{n X}{(n-1)(X-1)^{3} \sqrt{n-X}} \\
& \cdot\left\{F_{2}(X)-2(n-1) X(X-1) \cdot\{B-\phi(X)\}\{1-\nu(X)\}+3(X-1) f_{0}(X)\right\} \\
= & \frac{n X}{(n-1)(X-1)^{3} \sqrt{n-X}} \cdot\left\{G_{2}(X)+3(X-1) f_{0}(X)\right\} .
\end{aligned}
$$

Now, setting $x=1+t$, we obtain from (8.14) in [13]

$$
F_{2}(1+t)=t^{4}\left\{\frac{n\left(n^{2}-n+1\right) B}{6(n-1)}+O(t)\right\} .
$$

Since we have

$$
\begin{aligned}
& \phi(x)=x(n-x)^{n-1}, \quad \phi^{\prime}(x)=-n(x-1)(n-x)^{n-2}, \\
& \phi^{\prime \prime}(x)=n(n-1)(x-2)(n-x)^{n-3}, \\
& \phi^{\prime \prime \prime}(x)=-n(n-1)(n-2)(x-3)(n-x)^{n-4},
\end{aligned}
$$

we obtain immediately

$$
\psi(1+t)=B-\frac{n B}{2(n-1)} t^{2}+\frac{n(n-2) B}{3(n-1)^{2}} t^{3}+\cdots .
$$

Next, since we have easily

$$
(n-1-t)^{n-1}=B-B t+\frac{(n-2) B}{2(n-1)} t^{2}+\cdots,
$$

and 


$$
\begin{aligned}
(n-1-t)^{2 n-3 / 2}=(n-1)^{2 n-3 / 2}-\left(2 n-\frac{3}{2}\right)(n-1)^{2 n-5 / 2} t+ \\
\quad+\frac{1}{2}\left(2 n-\frac{3}{2}\right)\left(2 n-\frac{5}{2}\right)(n-1)^{2 n-7 / 2} t^{2}+\cdots \\
=(n-1)^{2 n-3 / 2}\left\{1-\frac{4 n-3}{2(n-1)} t+\frac{(4 n-3)(4 n-5)}{8(n-1)^{2}} t^{2}+\cdots,\right.
\end{aligned}
$$

we obtain from (2.4)

$$
\begin{aligned}
\nu(1+t)= & \sqrt{B} t^{2}(1+t)^{-2} \cdot(n-1)^{2 n-3 / 2}\left\{1-\frac{4 n-3}{2(n-1)} t+\frac{(4 n-3)(4 n-5)}{8(n-1)^{2}} t^{2} \cdots\right\} \\
& \times B^{-2} t^{-2}\left\{1-\frac{n-2}{2(n-1)} t+\cdots\right\}^{-2} \\
& \times B^{-1 / 2}\left\{n-1+t-\frac{n-2}{2(n-1)} t^{2}+\cdots\right\}^{-1 / 2} \\
= & 1-\frac{3 n-1}{n-1} t+\cdots .
\end{aligned}
$$

Thus, we obtain

$$
\begin{aligned}
G_{2}(1+t)= & F_{2}(1+t)-2(n-1)(1+t) t\{B-\phi(1+t)\}\{1-\nu(1+t)\} \\
= & B t^{4}\left\{\frac{n\left(n^{2}-n+1\right)}{6(n-1)}+O(t)\right\} \\
& -2(n-1)(1+t) t \cdot \frac{n B t^{2}}{2(n-1)}\left\{1-\frac{2(n-2)}{3(n-1)} t+\cdots\right\} \cdot \frac{(3 n-1) t}{n-1}\{1+\cdots\} \\
= & B t^{4}\left\{\frac{n\left(n^{2}-19 n+7\right)}{6(n-1)}+O(t)\right\}
\end{aligned}
$$

and by (8.17) in [13]

$$
\begin{aligned}
& G_{2}(1+t)+3 t f_{0}(1+t)=B t^{4}\left\{\frac{n\left(n^{2}-19 n+7\right)}{6(n-1)}+O(t)\right\} \\
& +B t^{4}\left\{\frac{n(2 n-1)}{2(n-1)}+O(t)\right\}=B t^{4}\left\{\frac{n\left(n^{2}-13 n+4\right)}{6(n-1)}+O(t)\right\} .
\end{aligned}
$$

Since $n^{2}-13 n+4>0$ for $n>(13+\sqrt{153}) / 2 \doteqdot 12.685$, we obtain $G_{2}(x)+3(x-1) f_{0}(x)$ is positive for $x>1$ sufficiently near $x=1$.

Q.E.D. 


\section{$\S 3$. Certain auxiliary inequalities (II).}

In this section, we shall investigate the sign of the function $G_{2}(x)+$ $3(x-1) f_{0}(x)$ for the interval $2 \leqq x<n$.

LEMMA 3.1. When $n \geqq 6, G_{2}(x)+3(x-1) f_{0}(x)$ is positive for $2 \leqq x<n$.

Proof. By (2.6) we see that $G_{2}(x)+3(x-1) f_{0}(x)$ is positive for $1<x<n$ if and only if

$$
\begin{gathered}
2(n-1) x(x-1)\{B-\psi(x)\} \nu(x)>2(n-1) x(x-1)\{B-\psi(x)\} \\
-F_{2}(x)-3(x-1) f_{0}(x) \quad \text { for } 1<x<n .
\end{gathered}
$$

Now, we rewrite the right hand side of (3.1) as follows.

$$
\begin{aligned}
& 2(n-1) x(x-1)\{B-\psi(x)\}-F_{2}(x)-3(x-1) f_{0}(x) \\
= & 2(n-1) x(x-1)\left\{B-x(n-x)^{n-1}\right\} \\
& +\left\{(2 n+1) x^{2}-2\left(2 n^{2}+5 n-4\right) x+16 n^{2}-16 n+3\right\} B \\
& -(n-x)^{n-1}\left\{-(n-1) x^{3}+\left(2 n^{2}-7 n+8\right) x^{2}+\left(4 n^{2}-13 n+3\right) x+3 n(2 n-1)\right\} \\
& -3(x-1)(2 n-1-x) B+3(n-x)^{n-1}(x-1)\left\{n-x+(n-1) x^{2}\right\} \\
= & -2(n-x)\{(2 n+1) x-(8 n-5)\} B-2(n-x)^{n}\left\{(n-1) x^{2}+(2 n-5) x+3 n\right\} .
\end{aligned}
$$

Hence, (3.1) is equivalent to

$$
\begin{gathered}
(n-1)(x-1)^{3}(n-x)^{2 n-5 / 2} \cdot \frac{\left\{B-x(n-x)^{n-1}\right\} \sqrt{B}}{x\left\{B-(n-x)^{n-1}\right\}^{2} \sqrt{n B-(n-x)^{n-1}}} \\
>-\{(2 n+1) x-(8 n-5)\} B-(n-x)^{n-1}\left\{(n-1) x^{2}+(2 n-5) x+3 n\right\} \\
\text { for } 1<x<n .
\end{gathered}
$$

We see easily that the left hand side is positive for $1<x<n$ and tends to zero as $x \rightarrow 1+0$ and the right hand side becomes zero at $x=1$.

On the right hand side we see that

$$
(2 n+1) x-(8 n-5) \geqq 0 \quad \text { for } x \geqq \frac{8 n-5}{2 n+1}=4-\frac{9}{2 n+1}
$$

and

$$
(n-1) x^{2}+(2 n-5) x+3 n>0 \quad \text { for }-\propto<x<+\infty,
$$

when $n>2$. Hence, the right hand side is negative for $x \geqq(8 n-5) /(2 n+1)$. Therefore (3.2) holds for $(8 n-5) /(2 n+1) \leqq x<n$.

Next, we investigate (3.2) for $1<x<(8 n-5) /(2 n+1)$. The condition that the 
right hand side of (3.2) is non positive is equivalent to

$$
\rho(x):=\frac{(n-x)^{n-1}\left\{(n-1) x^{2}+(2 n-5) x+3 n\right\}}{8 n-5-(2 n+1) x} \geqq B \quad \text { for } 1<x<\frac{8 n-5}{2 n+1} .
$$

We see easily that $\rho(1)=B$ and have

$$
\frac{d}{d x} \rho(x)=\frac{n(n-1)(x-1)^{2}(n-x)^{n-2}\{(2 n+1) x-2(n+5)\}}{\{8 n-5-(2 n+1) x\}^{2}} .
$$

Hence, we see that $\rho(x)$ is increasing in the interval

$$
\frac{2(n+5)}{2 n+1}<x<\frac{8 n-5}{2 n+1},
$$

when $n>5 / 2$. Furthermore, we have $2(n+5) /(2 n+1) \leqq 2$ if and only if $n \geqq 4$ and we have $(8 n-5) /(2 n+1) \geqq 2$ if and only if $n \geqq 7 / 4$.

In the following we suppose $n \geqq 4$, then $\rho(x)$ is increasing in the interval $2 \leqq x<(8 n-5) /(2 n+1)$.

Finally we estimate the value $\rho(2)$. From the definition of $\rho(x)$ we have

$$
\rho(2)=\frac{(n-2)^{n-1}(11 n-14)}{4 n-7}
$$

and

$$
\frac{\rho(2)}{B}=\left(\frac{n-2}{n-1}\right)^{n-1} \cdot \frac{11 n-14}{4 n-7}>1 \quad \text { for } n \geqq 6 \text {, }
$$

which will be proved in the next lemma. Then, we obtain

$$
\rho(x) \geqq \rho(2)>B \quad \text { for } 2 \leqq x<\frac{8 n-5}{2 n+1},
$$

and we see that the present lemma is true.

Q.E.D.

LEMMA 3.2. We have

$$
\left(\frac{n-2}{n-1}\right)^{n-1} \cdot \frac{11 n-14}{4 n-7}>1 \quad \text { for } n \geqq 6 .
$$

Proof. First of all, we show that the left hand side of the above inequality is increasing for $n>2$ as a function of $n$. We have

$$
\frac{d}{d n} \log \left\{\left(\frac{n-2}{n-1}\right)^{n-1} \cdot \frac{11 n-14}{4 n-7}\right\}=\log \frac{n-2}{n-1}+\frac{1}{n-2}-\frac{21}{(11 n-14)(4 n-7)} .
$$

Setting $t=1 /(n-1)$, the last expression of $n$ can be written as

$$
\log (1-t)+\frac{t}{1-t}-\frac{21}{44} \cdot t^{2} /\left(1-\frac{3 t}{11}\right)\left(1-\frac{3 t}{4}\right) \text {. }
$$


Since $0<t<1$, the above function of $t$ can be written in a power series of $t$ as

$$
\begin{aligned}
= & -\left(t+\frac{1}{2} t^{2}+\frac{1}{3} t^{3}+\cdots+\frac{1}{m} t^{m}+\cdots\right) \\
& +\left(t+t^{2}+t^{3}+\cdots+t^{m}+\cdots\right) \\
& -\frac{21}{44} t^{2}\left\{1+\frac{3}{11} t+\left(\frac{3}{11}\right)^{2} t^{2}+\cdots+\left(\frac{3}{11}\right)^{m} t^{m}+\cdots\right\} \\
& \quad \times\left\{1+\frac{3}{4} t+\left(\frac{3}{4}\right)^{2} t^{2}+\cdots+\left(\frac{3}{4}\right)^{m} t^{m}+\cdots\right\} \\
= & \left(\frac{1}{2} t^{2}+\frac{2}{3} t^{3}+\frac{3}{4} t^{4}+\cdots+\frac{m+1}{m+2} t^{m+2}+\cdots\right)-\frac{21}{44} t^{2} \sum_{m=0}^{\infty} a_{m} t^{m+2},
\end{aligned}
$$

where

$$
a_{m}=3^{m}\left(\frac{1}{4^{m}}+\frac{1}{4^{m-1} \cdot 11}+\cdots+\frac{1}{4 \cdot 11^{m-1}}+\frac{1}{11^{m}}\right), \quad m=0,1,2, \cdots .
$$

It the following we shall prove $(m+1) /(m+2)>(21 / 44) a_{m}, m=0,1,2, \cdots$. In fact, we have

$$
\begin{aligned}
& \frac{1}{2}-\frac{21}{44} a_{0}=\frac{1}{2}-\frac{21}{44}=\frac{1}{44}>0, \\
& \frac{2}{3}-\frac{21}{44} a_{1}=\frac{2}{3}-\frac{21}{44} \cdot \frac{3 \cdot 15}{44}=\frac{3872-2835}{3 \cdot 44 \cdot 44}=\frac{1037}{3 \cdot 44 \cdot 44}>0,
\end{aligned}
$$

and for $m \geqq 2$

$$
\begin{aligned}
& \frac{m+1}{m+2}-\frac{21}{44} a_{m}>\frac{m+1}{m+2}-\frac{1}{2} a_{m}>\frac{m+1}{m+2}-\frac{1}{2} \cdot\left(\frac{3}{4}\right)^{m}\left(1+\frac{1}{2}+\cdots+\frac{1}{2^{m}}\right) \\
& >\frac{m+1}{m+2}-\left(\frac{3}{4}\right)^{m}=\frac{m+1}{m+2} \cdot\left(\frac{3}{4}\right)^{m}\left\{\left(\frac{4}{3}\right)^{m}-\frac{m+2}{m+1}\right\} \\
& >\frac{m+1}{m+2} \cdot\left(\frac{3}{4}\right)^{m}\left\{1+\frac{m}{3}-\frac{m+2}{m+1}\right\}=\left(\frac{3}{4}\right)^{m} \cdot \frac{m^{2}+m-3}{3(m+2)}>0 .
\end{aligned}
$$

Thus we have proved

$$
\log (1-t)+\frac{t}{1-t}-\frac{21}{44} \cdot t^{2} /\left(1-\frac{3 t}{11}\right)\left(1-\frac{3 t}{4}\right)>0 \quad \text { for } 0<t<1
$$

and so $((n-2) /(n-1))^{n-1} \cdot(11 n-14) /(4 n-7)$ is increasing with respect $n$ for $n>2$.

Finally we have

$$
\left[\left(\frac{n-2}{n-1}\right)^{n-1} \cdot \frac{11 n-14}{4 n-7}\right]_{n=6}=\left(\frac{4}{5}\right)^{5} \cdot \frac{52}{17}=\frac{53248}{53125}>1
$$


and

$$
\left[\left(\frac{n-2}{n-1}\right)^{n-1} \cdot \frac{11 n-14}{4 n-7}\right]_{n=5}=\left(\frac{3}{4}\right)^{4} \cdot \frac{41}{13}=\frac{3321}{3328}<1 .
$$

From these we see that this lemma is true.

Q.E.D.

\section{$\S 4$. A property of the auxiliary function $\sigma(x)$.}

Using the arguments in the proof of Lemma 3.1, when $n>2$, the condition $G_{2}(x)+3(x-1) f_{0}(x)>0$ for $1<x \leqq 2$ is equivalent to

$$
\sigma(x)>B-\rho(x) \quad \text { for } 1<x \leqq 2,
$$

where

$$
\sigma(x):=\frac{(n-1) \sqrt{B(}(x-1)^{3}(n-x)^{n-5 / 2}\left\{B-x(n-x)^{n-1}\right\}}{x\{8 n-5-(2 n+1) x\}\left\{B-(n-x)^{n-1}\right\}^{2} \sqrt{n B-(n-x)^{n-1}}} .
$$

In this section, we shall prove that $\sigma(x)$ is monotone increasing in the interval $1<x \leqq 2$, when $n \geqq 6$.

LEMMA 4.1. The function $(x-1)(n-x)^{n-2} / x\{8 n-5-(2 n+1) x\}$ is monotone increasing in the interval $1 \leqq x \leqq 2$, when $n \geqq 3$.

Proof. We have

$$
\begin{aligned}
& \frac{d}{d x} \frac{(x-1)(n-x)^{n-2}}{x\{8 n-5-(2 n+1) x\}}=\frac{(n-x)^{n-3}}{x^{2}\{8 n-5-(2 n+1) x\}^{2}} \\
& \times\left\{n(8 n-5)+\left(4 n^{2}-31 n+15\right) x-\left(8 n^{2}-29 n+6\right) x^{2}+(n-3)(2 n+1) x^{3}\right\},
\end{aligned}
$$

the last factor of which is positive for $1<x<2$. In fact, its derivative with respect to $x$ is the quadratic polynomial of $x$ :

$$
4 n^{2}-31 n+15-2\left(8 n^{2}-29 n+6\right) x+3(n-3)(2 n+1) x^{2},
$$

whose values at $x=1$ and $x=2$ are $-6(n-1)^{2}<0$ and $-4 n^{2}+25 n-45<0$, respectively, and so whose value is negative for $1 \leqq x \leqq 2$. Therefore, the above cubic function of $x$ is decreasing in the interval $1 \leqq x \leqq 2$. Since, its value at $x=2$ is $9(n-2)>0$, it must be positive for $1 \leqq x \leqq 2$. Thus, we have proved that the given function in this lemma is increasing in the interval $1 \leqq x \leqq 2$, when $n \geqq 3$.

Q.E.D.

LEMMA 4.2. The function $(n-x)^{n-1 / 2}\left\{B-x(n-x)^{n-1}\right\}$ is monotone increasing in the interval $1 \leqq x \leqq 2$, when $n \geqq 6$.

Proof. For $0<x<n$, the derivative of this function is 


$$
\frac{1}{2}(n-x)^{n-3 / 2}\left[\{(4 n-1) x-2 n\}(n-x)^{n-1}-(2 n-1) B\right],
$$

the expression in the brackets of which is positive for $1<x \leqq 2$ as is shown in the following. Since we have

$$
\frac{d}{d x}\{(4 n-1) x-2 n\}(n-x)^{n-1}=n(n-x)^{n-2}\{3(2 n-1)-(4 n-1) x\}
$$

and

$$
1<\frac{3(2 n-1)}{4 n-1}<2
$$

$\{(4 n-1) x-2 n\}(n-x)^{n-1}$ takes its minimum in the interval $1 \leqq x \leqq 2$ at $x=1$ or $x=2$. And its value at $x=1$ and $x=2$ are $(2 n-1) B$ and $2(3 n-1)(n-2)^{n-1}$, respectively. Furthermore, we have

$$
2(3 n-1)(n-2)^{n-1}-(2 n-1) B=(2 n-1)(n-2)^{n-1}\left\{\frac{2(3 n-1)}{2 n-1}-\left(\frac{n-1}{n-2}\right)^{n-1}\right\},
$$

and we can prove the function $((n-1) /(n-2))^{n-1}$ of $n$ is decreasing for $n>2$.

In fact, we have

$$
\begin{aligned}
& \frac{d}{d n} \log \left(\frac{n-1}{n-2}\right)^{n-1}=\log \frac{n-1}{n-2}+(n-1)\left(\frac{1}{n-1}-\frac{1}{n-2}\right) \\
& =-\log \left(1-\frac{1}{n-1}\right)+1-1 /\left(1-\frac{1}{n-1}\right) \\
& =\left\{\frac{1}{n-1}+\frac{1}{2(n-1)^{2}}+\frac{1}{3(n-1)^{3}}+\cdots\right\}-\left\{\frac{1}{n-1}+\frac{1}{(n-1)^{2}}+\frac{1}{(n-1)^{3}}+\cdots\right\}<0 .
\end{aligned}
$$

Using this fact, we have for $n \geqq 7$

$$
\frac{2(3 n-1)}{2 n-1}-\left(\frac{n-1}{n-2}\right)^{n-1}>3-\left(\frac{6}{5}\right)^{6}=3-\frac{46656}{15625}=\frac{219}{15625}>0
$$

and for $6 \leqq n<7$

$$
\frac{2(3 n-1)}{2 n-1}-\left(\frac{n-1}{n-2}\right)^{n-1}>\frac{40}{13}-\left(\frac{5}{4}\right)^{5}=\frac{40}{13}-\frac{3125}{1024}=\frac{335}{13312}>0,
$$

and

$$
\left[\frac{2(3 n-1)}{2 n-1}-\left(\frac{n-1}{n-2}\right)^{n-1}\right]_{n=5}=\frac{28}{9}-\left(\frac{4}{3}\right)^{4}=-\frac{4}{81}<0 .
$$

Thus, we have proved that

$$
\{(4 n-1) x-2 n\}(n-x)^{n-1}>(2 n-1) B \quad \text { for } 1<x<2,
$$

when $n \geqq 6$, which implies this lemma.

Q.E.D. 
LEMMA 4.3. The function $\left(B-(n-x)^{n-1}\right) /(x-1)$ is monotone decreasing in the interval $1<x<n$, when $n>2$.

Proof. For $1<x<n$, the derivative of this function is

$$
\frac{(n-x)^{n-2}\{(n-2) x+1\}-B}{(x-1)^{2}} \text {. }
$$

Since we have

$$
\frac{d}{d x}(n-x)^{n-2}\{(n-2) x+1\}=-(n-1)(n-2)(n-x)^{n-3}<0
$$

for $1<x<n$, we obtain

$$
(n-x)^{n-2}\{(n-2) x+1\}-B<(n-1)^{n-1}-B=0 \quad \text { for } 1<x<n,
$$

which implies this lemma.

Q.E.D.

LEMMA 4.4. The function $\left\{\left(B-(n-x)^{n-1}\right) /(x-1)\right\} \cdot\left\{n B-(n-x)^{n-1}\right\}$ is monotone decreasing in the interval $1<x<n$, when $n \geqq 4$.

Proof. For $1<x<n$, the derivative of this function is $p(x) /(x-1)^{2}$, where

$$
\begin{aligned}
p(x):= & \left\{(n-x)^{n-2}((n-2) x+1)-B\right\}\left\{n B-(n-x)^{n-1}\right\} \\
& +(n-1)(x-1)(n-x)^{n-2}\left\{B-(n-x)^{n-1}\right\} .
\end{aligned}
$$

Since we have

$$
p^{\prime}(x)=(n-1)(x-1)(n-x)^{n-3}\left\{2(2 n-3)(n-x)^{n-1}-(n-2)(n+1) B\right\}
$$

and for $1<x<n$

$$
\begin{gathered}
2(2 n-3)(n-x)^{n-1}-(n-2)(n+1) B<\left[2(2 n-3)(n-x)^{n-1}-(n-2)(n+1) B\right]_{x=1} \\
=-\left(n^{2}-5 n+4\right) B=-(n-1)(n-4) B \leqq 0,
\end{gathered}
$$

when $n \geqq 4$, we obtain $p^{\prime}(x)<0$ for $1<x<n$, which implies this lemma. Q.E.D.

Proposition 2. The function $\sigma(x)$ defined by (4.2) is monotone increasing in the interval $1<x<2$, when $n \geqq 6$.

Proof. First we factorize $\sigma(x)$ as

$$
\begin{aligned}
\sigma(x)= & (n-1) \sqrt{B} \times \frac{(x-1)(n-x)^{n-2}}{x\{8 n-5-(2 n+1)\}} \times(n-x)^{n-1 / 2}\left\{B-x(n-x)^{n-1}\right\} \\
& \times\left\{\frac{B-(n-x)^{n-1}}{x-1}\right\}^{-3 / 2} \times\left\{\frac{B-(n-x)^{n-1}}{x-1} \cdot\left\{n B-(n-x)^{n-1}\right\}\right\}^{-1 / 2} .
\end{aligned}
$$


By means of Lemma 4.1-Lemma 4.4, we see that $\sigma(x)$ is increasing in the interval $1<x<2$, when $n \geqq 6$.

Q.E.D.

\section{$\S 5$. The inequality $\sigma(x)>B-\rho(x)$.}

In this section we shall prove that $G_{2}(x)+3(x-1) f_{0}(x)$ is positive for $(2 n+10) /(2 n+1) \leqq x<2$.

LEMMA 5.1. We have

$$
\rho(x)-B>-\frac{n(n+2) B(x-1)^{3}\{2 n+13-(2 n+1) x\}}{36(n-1)(2 n+1)(2 n-5)} \quad \text { for } 1<x \leqq \frac{2 n+10}{2 n+1},
$$

when $n \geqq 3$.

Proof. When $n \geqq 3$, from (3.3) and (3.4) we have

$$
\begin{aligned}
\rho(x)-B & =\rho(x)-\rho(1)=\int_{1}^{x} \rho^{\prime}(t) d t \\
& =-n(n-1) \int_{1}^{x} \frac{(t-1)^{2}(n-t)^{n-2}\{2 n+10-(2 n+1) t\} d t}{\{8 n-5-(2 n+1) t\}^{2}} \\
& >-n(n-1)^{n-2} \int_{1}^{x} \frac{n-t}{\{8 n-5-(2 n+1) t\}^{2}} \cdot(t-1)^{2}\{2 n+10-(2 n+1) t\} d t
\end{aligned}
$$

for $1<x<(2 n+10) /(2 n+1)$. In this interval the function $(n-x) /\{8 n-5-(2 n+1) x\}^{2}$ is increasing, because

$$
\frac{d}{d x} \frac{n-x}{\{8 n-5-(2 n+1) x\}^{2}}=\frac{4 n^{2}-6 n+5-(2 n+1) x}{\{8 n-5-(2 n+1) x\}^{3}}
$$

and

$$
\begin{aligned}
4 n^{2}-6 n+5-(2 n+1) x & \geqq 4 n^{2}-6 n+5-(2 n+1) \cdot \frac{2 n+10}{2 n+1} \\
& =(2 n+1)(2 n-5)>0 .
\end{aligned}
$$

Therefore, we obtain

$$
\begin{aligned}
\frac{n-t}{8 n-5-(2 n+1) t^{2}} & <\frac{n-\frac{2 n+10}{2 n+1}}{\{8 n-5-(2 n+10)\}^{2}}=\frac{2 n^{2}-n-10}{9(2 n+1)(2 n-5)^{2}} \\
& =\frac{n+2}{9(2 n+1)(2 n-5)} \quad \text { for } 1<t<x \leqq \frac{2 n+10}{2 n+1} .
\end{aligned}
$$

Using this inequality, we obtain

$$
\rho(x)-B>-\frac{n(n+2)(n-1)^{n-2}}{9(2 n+1)(2 n-5)} \int_{1}^{x}(t-1)^{2}\{2 n+10-(2 n+1) t\} d t
$$




$$
\begin{aligned}
& =-\frac{n(n+2) B}{9(n-1)(2 n+1)(2 n-5)} \int_{0}^{x-1} t^{2}\{9-(2 n+1) t\} d t \\
& =-\frac{n(n+2) B(x-1)^{3}\{2 n+13-(2 n+1) x\}}{36(n-1)(2 n+1)(2 n-5)} \text { for } 1<x \leqq \frac{2 n+10}{2 n+1} . \\
& \text { Q.E. D. }
\end{aligned}
$$

LEMMA 5.2. We have

$$
\sigma(x)>\frac{n \sqrt{n-1}}{36 B^{2}} \cdot \frac{n+2}{n+5}\left\{\frac{(n+2)(2 n-5)}{2 n+1}\right\}^{2 n-13 / 2}(x-1)^{3}(3 n-1-2 x)
$$

for $1<x \leqq(2 n+10) /(2 n+1)$, when $n \geqq 25 / 4$.

Proof. As in the proof of Proposition 2, we represent $\sigma(x)$ as a product:

$$
\begin{aligned}
& \frac{\sigma(x)}{\sqrt{n-1} B}=\frac{(x-1)(n-x)^{n-2}}{x\{8 n-5-(2 n+1) x\}} \times(n-x)^{n-1 / 2}\left\{B-x(n-x)^{n-1}\right\} \\
& \times\left\{\frac{B-(n-x)^{n-1}}{x-1}\right\}^{-3 / 2} \times\left\{\frac{B-(n-x)^{n-1}}{x-1} \cdot\left\{n B-(n-x)^{n-1}\right\}\right\}^{-1 / 2} .
\end{aligned}
$$

First of all, we estimate the first factor. We see easily

$$
\frac{2 n+10}{2 n+1} \leqq \frac{8 n-5}{2(2 n+1)} \quad \text { when } n \geqq \frac{25}{4} .
$$

Therefore, assuming $n \geqq 25 / 4$, we have

$$
6(n-1)<x\{8 n-5-(2 n+1) x\}<\frac{2 n+10}{2 n+1} \cdot(6 n-15)=\frac{6(n+5)(2 n-5)}{2 n+1}
$$

for $1<x<(2 n+10) /(2 n+1)$ and hence

$$
\frac{(x-1)(n-x)^{n-2}}{x\{8 n-5-(2 n+1) x\}}>\left(n-\frac{2 n+10}{2 n+1}\right)^{n-2}(x-1) / \frac{6(n+5)(2 n-5)}{2 n+1}
$$

i. e.

$$
\frac{(x-1)(n-x)^{n-2}}{x\{8 n-5-(2 n+1) x\}}>\frac{(n+2)^{n-2}(2 n-5)^{n-3}}{6(n+5)(2 n+1)^{n-3}} \cdot(x-1) \text { for } 1<x<\frac{2 n+10}{2 n+1},
$$

when $n \geqq 25 / 4$.

Second, we estimate the second factor as follows. Assuming $n \geqq 3$, we have for $1<x<(2 n+10) /(2 n+1)$

$$
\begin{aligned}
& B-x(n-x)^{n-1}=\psi(1)-\psi(x)=\int_{x}^{1} \psi^{\prime}(t) d t \\
& =n \int_{1}^{x}(t-1)(n-t)^{n-2} d t=n \int_{1}^{x}(n-t)^{n-3}(t-1)(n-t) d t
\end{aligned}
$$




$$
\begin{aligned}
& >n\left(n-\frac{2 n+10}{2 n+1}\right)^{n-3} \int_{1}^{x}(t-1)(n-t) d t \\
& =\frac{n}{6}\left\{\frac{(n+2)(2 n-5)}{2 n+1}\right\}^{n-3}(x-1)^{2}(3 n-1-2 x) .
\end{aligned}
$$

Hence, we obtain

$$
(n-x)^{n-1 / 2}\left\{B-x(n-x)^{n-1}\right\}>\frac{n}{6}\left\{\frac{(n+2)(2 n-5)}{2 n+1}\right\}^{2 n-7 / 2}(x-1)^{2}(3 n-1-2 x)
$$

for $1<x<(2 n+10) /(2 n+1)$, when $n \geqq 3$.

Third, we estimate the third and fourth factors by Lemma 4.3 and Lemma 4.4 as follows:

$$
\left\{\frac{B-(n-x)^{n-1}}{x-1}\right\}^{-3 / 2}>\left\{(n-1)^{n-1}\right\}^{-3 / 2}=B^{-3 / 2}
$$

and

$$
\left\{\frac{B-(n-x)^{n-1}}{x-1} \cdot\left\{n B-(n-x)^{n-1}\right\}\right\}^{-1 / 2}>\{B \cdot(n-1) B\}^{-1 / 2}=(n-1)^{-1 / 2} B^{-1}
$$

for $1<x<n$, when $n \geqq 4$.

Thus, by means of (5.3), (5.4) and the above two inequalities we obtain

$$
\begin{aligned}
\sigma(x)> & \sqrt{n-1} \sqrt{B} \times \frac{(n+2)^{n-2}(2 n-5)^{n-3}(x-1)}{6(n+5)(2 n+1)^{n-3}} \\
& \times \frac{n}{6}\left\{\frac{(n+2)(2 n-5)}{2 n+1}\right\}^{2 n-7 / 2}(x-1)^{2}(3 n-1-2 x) \times B^{-3 / 2} \times(n-1)^{-1 / 2} B^{-1} \\
= & \frac{n \sqrt{n-1}}{36 B^{2}} \cdot \frac{n+2}{n+5}\left\{\frac{(n+2)(2 n-5)}{2 n+1}\right\}^{3 n-13 / 2}(x-1)^{3}(3 n-1-2 x)
\end{aligned}
$$

for $1<x<(2 n+10) /(2 n+1)$, when $n \geqq 25 / 4$.

Q.E.D.

LEMMA 5.3. When $n \geqq 13$, we have $\sigma(x)>B-\rho(x)$ for $(2 n+10) /(2 n+1) \leqq x<2$.

Proof. $\sigma(x)$ is positive for $1<x<2$ by Proposition 2 , when $n \geqq 6$. And $\rho(x)-B$ is negative for $1<x<(2 n+10) /(2 n+1)$ by (3.4), when $n \geqq 5 / 2$.

Assuming $n \geqq 25 / 4$ in the following, by means of Lemma 5.1 and Lemma 5.2 we have

$$
\begin{aligned}
& \frac{B-\rho(x)}{\sigma(x)}<\left[\frac{n(n+2) B(x-1)^{3}\{2 n+13-(2 n+1) x\}}{36(n-1)(2 n+1)(2 n-5)}\right] \\
& \quad /\left[\frac{n \sqrt{n-1}}{36 B^{2}} \cdot \frac{n+2}{n+5}\left\{\frac{(n+2)(2 n-5)}{2 n+1}\right\}^{3 n-13 / 2}(x-1)^{3}(3 n-1-2 x)\right] \\
& =\frac{B^{3}(n+5)}{(n-1)^{3 / 2}(2 n+1)(2 n-5)} \cdot\left\{\frac{2 n+1}{(n+2)(2 n-5)}\right\}^{3 n-13 / 2} \cdot \frac{2 n+13-(2 n+1) x}{3 n-1-2 x},
\end{aligned}
$$


i.e.

$$
\frac{B-\rho(x)}{\sigma(x)}<\frac{(n+5)(n-1)^{2}}{(2 n+1)(2 n-5)} \cdot\left\{\frac{(n-1)(2 n+1)}{(n+2)(2 n-5)}\right\}^{3 n-13 / 2} \cdot \frac{2 n+13-(2 n+1) x}{3 n-1-2 x}
$$

for $1<x \leqq(2 n+10) /(2 n+1)$. Since we have $(2 n+13-(2 n+1) x) /(3 n-1-2 x)=$ $(2 n+1) /\left(2 n^{2}-n-7\right)$ at $x=(2 n+10) /(2 n+1)$, we obtain from $(5.5)$

$$
B-\rho\left(\frac{2 n+10}{2 n+1}\right) / \sigma\left(\frac{2 n+10}{2 n+1}\right)<b_{n} c_{n}
$$

where

$$
\begin{aligned}
& b_{n}:=\frac{4(n+5)(n-1)}{(2 n+1)(2 n-5)} \cdot\left\{\frac{(n-1)(2 n+1)}{(n+2)(2 n-5)}\right\}^{3 n-13 / 2}, \\
& c_{n}:=\frac{2 n^{2}-n-1}{4\left(2 n^{2}-n-7\right)}=\frac{1}{4}\left(1+\frac{6}{2 n^{2}-n-7}\right) .
\end{aligned}
$$

We see easily that

$$
c_{n}>\frac{1}{4} \quad \text { for } n>\frac{1+\sqrt{57}}{4} \risingdotseq \frac{1+7.549}{4}=2.13725
$$

and $c_{n} \downarrow 1 / 4$ as $n \rightarrow \infty$. We shall prove in the next lemma that for $n \geqq 8 b_{n} \downarrow 1$ as $n \rightarrow \infty$. Here, supposing this fact, we have

$$
\begin{aligned}
b_{12} c_{12} & =\frac{4 \cdot 17 \cdot 11}{25 \cdot 19} \cdot\left(\frac{11 \cdot 25}{14 \cdot 19}\right)^{29.5} \times \frac{1}{4} \cdot \frac{275}{269} \\
& =\frac{17 \cdot 11 \cdot 11}{19 \cdot 269} \cdot\left(\frac{275}{269}\right)^{29.5} \risingdotseq \frac{2057}{5111} \times(1.0338345)^{29.5} \\
& =0.4024652 \times 2.66874=1.074075>1
\end{aligned}
$$

and

$$
\begin{aligned}
b_{13} c_{13} & =\frac{4 \cdot 18 \cdot 12}{27 \cdot 21} \cdot\left(\frac{12 \cdot 27}{15 \cdot 21}\right)^{32.5} \times \frac{1}{4} \cdot \frac{324}{318} \\
& =\frac{8 \cdot 18}{7 \cdot 53} \times\left(\frac{36}{35}\right)^{32.5} \risingdotseq \frac{144}{371} \times(1.0285714)^{32.5} \\
& =0.3881401 \times 2.49816=0.969636<1 .
\end{aligned}
$$

Therefore, it must be

$$
b_{n} c_{n}<1 \quad \text { for } n \geqq 13 .
$$

Hence, we obtain from (5.6) and (5.9)

$$
\sigma\left(\frac{2 n+10}{2 n+1}\right)>B-\rho\left(\frac{2 n+10}{2 n+1}\right), \quad \text { when } n \geqq 13 .
$$

On the other hand, we see that $B-\rho(x)$ is decreasing in the interval 
$(2 n+10) /(2 n+1) \leqq x \leqq 2$, when $n \geqq 4$, by (3.4) and $\sigma(x)$ is increasing in the interval $1<x<2$, when $n \geqq 6$, by Proposition 2. Hence, we obtain from these facts and (5.10)

$$
\sigma(x)>B-\rho(x) \quad \text { for } \frac{2 n+10}{2 n+1} \leqq x<2 \text {, when } n \geqq 13 \text {. Q. E. D. }
$$

LEMMA 5.4. $b_{n}$ defined by (5.7) is monotone decreasing with respect to $n$ for $n \geqq 8$ and tends to 1 as $n \rightarrow \infty$.

Proof. We have from (5.7)

$$
b_{n}=4 \cdot\left[\frac{(n+5)^{2}(n+2)^{7}(2 n-5)^{5}}{(n-1)^{5}(2 n+1)^{9}}\right]^{1 / 2} \cdot\left[\left\{\frac{(n-1)(2 n+1)}{(n+2)(2 n-5)}\right\}^{n-1}\right]^{3} .
$$

In the following, we shall show that the both functions of $n$ in the above pairs of brackets are decreasing for $n \geqq 8$.

First, we have

$$
\begin{gathered}
\frac{d}{d n} \frac{(n+5)^{2}(n+2)^{7}(2 n-5)^{5}}{(n-1)^{5}(2 n+1)^{9}}=-\frac{(n+5)(n+2)^{6}(2 n-5)^{4}}{(n-1)^{6}(2 n+1)^{10}} \\
\quad \times\left(48 n^{3}-216 n^{2}-1197 n+555\right)
\end{gathered}
$$

and

$$
48 n^{3}-216 n^{2}-1197 n+555>0 \quad \text { for } n \geqq 8 \text {. }
$$

Therefore, we see that $\frac{(n+5)^{2}(n+2)^{7}(2 n-5)^{5}}{(n-1)^{5}(2 n+1)^{9}}$ is decreasing for $n \geqq 8$ and

$$
\lim _{n \rightarrow \infty} \frac{(n+5)^{2}(n+2)^{7}(2 n-5)^{5}}{(n-1)^{5}(2 n+1)^{9}}=\frac{1}{16} .
$$

Second, setting $1 /(n-1)=t$, we have

$$
\left\{\frac{(n-1)(2 n+1)}{(n+2)(2 n-5)}\right\}^{n-1}=\left\{\frac{2+3 t}{(1+3 t)(2-3 t)}\right\}^{1 / t} .
$$

We show that the function of $t$ of the right hand side is increasing with respect to $t$ for $0<t<2 / 3$.

$$
\begin{gathered}
\frac{d}{d t}\left\{\frac{1}{t} \log \frac{2+3 t}{(1+3 t)(2-3 t)}\right\}=-\frac{1}{t^{2}} \log \frac{2+3 t}{(1+3 t)(2-3 t)} \\
+\frac{3}{t}\left\{\frac{1}{2+3 t}-\frac{1}{1+3 t}+\frac{1}{2-3 t}\right\} \\
=\frac{1}{t^{2}} \log \left(1-\frac{9 t^{2}}{2+3 t}\right)+\frac{9(4+3 t)}{(1+3 t)(2+3 t)(2-3 t)} .
\end{gathered}
$$

For $t>0$, the condition $9 t^{2} /(2+3 t)<1$ is equivalent to $9 t^{2}-3 t-2=(3 t-2)(3 t+1)<0$, 
i. e. $t<2 / 3$, which is equivalent to $n>5 / 2$. Then, we have

$$
\begin{aligned}
& \frac{1}{t^{2}} \log \left(1-\frac{9 t^{2}}{2+3 t}\right)=-\frac{1}{t^{2}} \sum_{m=1}^{\infty} \frac{1}{m}\left(\frac{9 t^{2}}{2+3 t}\right)^{m} \\
& >-\frac{9}{2+3 t}-\frac{81 t^{2}}{2(2+3 t)^{2}} \sum_{m=0}^{\infty}\left(\frac{9 t^{2}}{2+3 t}\right)^{m}=-\frac{9}{2+3 t}-\frac{81 t^{2}}{2(2+3 t)^{2}} \\
& \times\left\{1 /\left(1-\frac{9 t^{2}}{2+3 t}\right)\right\}=-\frac{9}{2+3 t}-\frac{81 t^{2}}{2(2+3 t)\left(2+3 t-9 t^{2}\right)} \\
& =-\frac{9\left(4+6 t-9 t^{2}\right)}{2(1+3 t)(2+3 t)(2-3 t)}
\end{aligned}
$$

and hence

$$
\begin{aligned}
\frac{1}{t^{2}} \log \left(1-\frac{9 t^{2}}{2+3 t}\right) & +\frac{9(4+3 t)}{(1+3 t)(2+3 t)(2-3 t)} \\
& >\frac{9\left(4+9 t^{2}\right)}{2(1+3 t)(2+3 t)(2-3 t)}>0 .
\end{aligned}
$$

Therefore, $\left\{\frac{2+3 t}{(1+3 t)(2-3 t)}\right\}^{1 / t}$ is increasing with respect to $t$ for $0<t<2 / 3$ and

$$
\begin{aligned}
& \lim _{t \rightarrow 0}\left\{\frac{2+3 t}{(1+3 t)(2-3 t)}\right\}^{1 / t}=\lim _{n \rightarrow \infty}\left\{\frac{n-1}{n+2} \cdot \frac{2 n+1}{2 n-5}\right\}^{n-1} \\
= & \lim _{n \rightarrow \infty}\left(\frac{1}{1+3 /(n-1)}\right)^{n-1} \cdot \lim \left[\left(1+\frac{6}{2 n-5}\right)^{(2 n-5) / 6}\right]^{6(n-1) /(2 n-5)}=\frac{1}{e^{3}} \cdot e^{3}=1 .
\end{aligned}
$$

Thus, we have proved that $\left\{\frac{(n-1)(2 n+1)}{(n+2)(2 n-5)}\right\}^{n-1}$ is decreasing for $n>5 / 2$ and tends to 1 as $n \rightarrow \infty$.

Q.E.D.

PROPOSITION 3. When $n \geqq 13$, we have

$$
G_{2}(x)+3(x-1) f_{0}(x)>0 \quad \text { for } \frac{2 n+10}{2 n+1} \leqq x<n .
$$

Proof. By Lemma 3.1, this inequality is true for $2 \leqq x<n$ and it is also true for $(2 n+10) /(2 n+1) \leqq x<2$ by (4.1) and Lemma 5.3.

Q.E.D.

\section{$\S 6$. Evaluations of some constants.}

Setting

$$
\alpha_{0}=\alpha_{0}(n):=X_{n}{ }^{-1}(2), \quad \alpha_{1}=\alpha_{1}(n):=X_{n}{ }^{-1}\left(\frac{2 n+10}{2 n+1}\right),
$$


we shall evaluate $\alpha_{0}$ and $\alpha_{1}$ in this section.

LEMMA 6.1. When $n \geqq 6$, we have

$$
\frac{1}{3}<\alpha_{0}(n)<\frac{5}{12}=0.41 \dot{6} .
$$

Proof. In order to prove the inequality $a<\alpha_{0}(n)$ for $0<a<1$, it is sufficient to prove $a(n-a)^{n-1}<2(n-2)^{n-1}$, i. e.

$$
\left(\frac{n-a}{n-2}\right)^{n-1}<\frac{2}{a}
$$

For the constant $a$, the function of $n$ of the left hand side of (6.3) is decreasing, because

$$
\begin{aligned}
& \frac{d}{d n}\left\{\log \left(\frac{n-a}{n-2}\right)^{n-1}\right\}=\log \frac{n-a}{n-2}-\left(\frac{1-a}{n-a}+\frac{1}{n-2}\right) \\
& =-u\left[\frac{1-a}{1-a u}+\frac{1}{1-2 u}-\frac{1}{u}\{\log (1-a u)-\log (1-2 u)\}\right],
\end{aligned}
$$

where $u=1 / n$, and

$$
\begin{aligned}
& \frac{1-a}{1-a u}+\frac{1}{1-2 u}-\frac{1}{u} \log (1-a u)+\frac{1}{u} \log (1-2 u) \\
& =\sum_{m=1}^{\infty}\left\{\left(1-\frac{m}{m+1} a\right) a^{m}+\frac{m-1}{m+1} 2^{m}\right\} u^{m}>0 .
\end{aligned}
$$

Hence, if $((6-a) / 4)^{5}<2 / a$, then $(6.3)$ holds for $n \geqq 6$. Now, $((6-a) / 4)^{5}<2 / a$ is equivalent to $a(6-a)^{5}<2048$ and for $a=1 / 3$ we have

$$
a(6-a)^{5}=\frac{17^{5}}{3^{6}}=\frac{1419857}{729} \doteqdot 1947.678 .
$$

Hence it must be $\alpha_{0}(n)>1 / 3$.

Next, in order to prove the inequality $\alpha_{0}(n)<b$ for $0<b<1$, it is sufficient to prove

$$
\left(\frac{n-b}{n-2}\right)^{n-1}>\frac{2}{b}
$$

Since we have

$$
\lim _{n \rightarrow \infty}\left(\frac{n-b}{n-2}\right)^{n-1}=\lim _{n \rightarrow \infty}\left(1+\frac{2-b}{n-2}\right)^{n-1}=e^{2-2}
$$

(6.4) holds for $n>2$, provided $e^{2-b} \geqq 2 / b$, i.e.

$$
e^{b} \leqq \frac{e^{2}}{2} b .
$$


Now, for $b=5 / 12$ we have

$$
e^{b} \doteqdot 1.517, \quad \frac{e^{2}}{2} b=\frac{5}{24} e^{2} \doteqdot 1.539,
$$

Hence (6.4) holds for $n>2$ and $b=5 / 12$, and it must be $\alpha_{0}(n)<5 / 12$. Q. E. D.

Remark. (6.5) does not hold for $b=2 / 5=0.4$. In fact, $2^{b}=e^{2 / 5} \doteqdot 1.492,\left(e^{2} / 2\right) b$ $=(2 / 10) e^{2} \doteqdot 1.479$.

LEMMA 6.2. When $n>2$, we have

$$
x>2-X_{n}(x) \quad \text { for } 0<x<1 \text {. }
$$

Proof. By virtue of Lemma 2.1, it is sufficient to prove

$$
\left(\frac{n-X}{n-1}\right)^{n-1}>2-X \quad \text { for } 1<x<n .
$$

Since we have

$$
\frac{d}{d X}\left[\left(\frac{n-X}{n-1}\right)^{n-1}+X\right]=1-\left(\frac{n-X}{n-1}\right)^{n-2}>0,
$$

we obtain easily (6.7).

Q.E.D.

LEMMA 6.3. When $n \geqq 10$, we have

$$
\frac{2 n-8}{2 n+1}<\alpha_{1}(n)=X_{n}{ }^{-1}\left(\frac{2 n+10}{2 n+1}\right)<\frac{2 n-6}{2 n+1} \text {. }
$$

Proof. The left side inequality is evident by Lemma 6.2 .

The right side inequality is equivalent to

$$
\frac{2 n-6}{2 n+1} \cdot\left(n-\frac{2 n-6}{2 n+1}\right)^{n-1}>\frac{2 n+10}{2 n+1} \cdot\left(n-\frac{2 n+10}{2 n+1}\right)^{n-1},
$$

that is

$$
\left(\frac{2 n^{2}-n+6}{2 n^{2}-n-10}\right)^{n-1}>\frac{n+5}{n-3} .
$$

Now, since we have

$$
\begin{aligned}
& \left(\frac{2 n^{2}-n+6}{2 n^{2}-n-10}\right)^{n-1}=\left(1+\frac{16}{2 n^{2}-n-10}\right)^{n-1} \\
& >1+\frac{16(n-1)}{2 n^{2}-n-10}+\frac{8 \cdot 16 \cdot(n-1)(n-2)}{\left(2 n^{2}-n-10\right)^{2}}+\frac{8 \cdot 16^{2} \cdot(n-1)(n-2)(n-3)}{3\left(2 n^{2}-n-10\right)^{3}},
\end{aligned}
$$

(6.9) is implied from the following inequality: 


$$
\frac{2(n-1)}{2 n^{2}-n-10}+\frac{16(n-1)(n-2)}{\left(2 n^{2}-n-10\right)^{2}}+\frac{16^{2}(n-1)(n-2)(n-3)}{3\left(2 n^{2}-n-10\right)^{3}} \geqq \frac{1}{n-3},
$$

which is equivalent to

$$
12 n^{5}-92 n^{4}-813 n^{3}+6908 n^{2}-16116 n+12288 \geqq 0 .
$$

First, we show that (6.10) holds for $n \geqq 13$. In fact, we have for $n \geqq 13$

$$
12 n^{2}-92 n-813 \geqq 12 \cdot 13^{2}-92 \cdot 13-813=19
$$

and

$$
19 n^{3}+6908 n^{2}-16116 n+12288>0 .
$$

Second, for $12 \leqq n<13$, we have

$$
12 n^{2}-92 n-813 \geqq 12 \cdot 12^{2}-92 \cdot 12-813=-189
$$

and

$$
\begin{aligned}
& -189 n^{3}+6908 n^{2}-16116 n+12288 \\
& \geqq n\left(-189 \cdot 12^{2}+6908 \cdot 12-16116\right)+12288=39564 n+12288>0,
\end{aligned}
$$

which implies (6.10) for $12 \leqq n<13$.

Third, for $11 \leqq n<12$, we have

$$
12 n^{2}-92 n-813 \geqq 12 \cdot 11^{2}-92 \cdot 11-813=-373
$$

and

$$
\begin{aligned}
& -373 n^{3}+6908 n^{2}-16116 n+12288 \\
& >n\left(-373 \cdot 12^{2}+6908 \cdot 12-16116\right)+12288=13068 n+12288>0,
\end{aligned}
$$

since $6908 / 2 \times 373 \doteqdot 9.260<11$. Therefore $(6.10)$ holds also for $11 \leqq n<12$.

Fourth, for $10.2 \leqq n<11$, we have

$$
12 n^{2}-92 n-813 \geqq 12 \cdot 10.2^{2}-92 \cdot 10.2-813=-502.92
$$

and

$$
\begin{aligned}
& -502.92 n^{3}+6908 n^{2}-16116 n+12288 \\
& >n\left(-502.92 \cdot 11^{2}+6908 \cdot 11-16116\right)+12288=-981.32 n+12288>0
\end{aligned}
$$

since $6908 / 2 \times 502.92 \doteqdot 6.868<10.2$. Therefore $(6.10)$ holds also for $10.2 \leqq n<11$.

Last, for $10 \leqq n<10.2$, we have

$$
12 n^{2}-92 n-813 \geqq-533
$$

and

$$
\begin{aligned}
& -533 n^{3}+6908 n^{2}-16116+12288 \\
& >n\left(-533 \cdot 10.2^{2}+6908 \cdot 10.2-16116\right)+12288 \\
& =-1107.72 n+12288>0
\end{aligned}
$$


since $6908 / 2 \times 533 \doteqdot 6.480<10$. Therefore $(6.10)$ holds also for $10 \leqq n<10.2$. $n \geqq 10$.

Thus, we have proved that (6.10) is true for $n \geqq 10$. Hence (6.9) holds for

Remark. We check (6.9) for $n=9$.

$$
\left(\frac{2 n^{2}-n+6}{2 n^{2}-n-10}\right)^{n-1}=\left(\frac{159}{143}\right)^{8} \doteqdot 2.336, \quad \frac{n+5}{n-3}=\frac{14}{6} \doteqdot 2.333,
$$

which shows that (6.9) may be true for $9 \leqq n<10$. But the method taken in Lemma 6.3 does not go well. Next, for $n=8$ we have

$$
\left(\frac{2 n^{2}-n+6}{2 n^{2}-n-10}\right)^{n-1}=\left(\frac{126}{110}\right)^{7} \doteqdot 2.587, \quad \frac{n+5}{n-3}=\frac{13}{5}=2.600,
$$

which shows (6.9) is not true for $n=8$.

$\S 7$. Evaluations of $U_{0}(x)$ and $U_{4}(x)$ for $\alpha_{1}(n) \leqq x<1$.

In the following two sections, we shall evaluate the functions $U_{i}(x), \imath=0,1$, $2,4,5,6$, defined in $\S 1$ and appeared in $V\left(x, X_{n}(x)\right)$, for the interval $\alpha_{1}(n) \leqq x<1$, considering an effect of Proposition 3.

In the following, we set for simplicity

$$
\begin{gathered}
t=1-x, \quad s=X_{n}(x)-1 \quad \text { for } 0<x<1 \\
t_{0}=1-\alpha_{1}(n), \quad s_{0}=\frac{2 n+10}{2 n+1}-1=\frac{9}{2 n+1}
\end{gathered}
$$

and we obtain by Lemma 6.2 and Lemma 6.3 the inequalities

$$
\begin{aligned}
0 & <t<s, \\
\frac{7}{2 n+1} & <t_{0}<\frac{9}{2 n+1} .
\end{aligned}
$$

LEMMA 7.1. When $n \geqq 10$, we have

$$
\begin{aligned}
U_{0}(x) & >\frac{16 B}{3} \cdot \frac{n(n-1)^{3}(n-4)^{2}\left(n^{2}-n+1\right)(2 n+1)^{3 / 2}\left(2 n^{2}-n+8\right)^{1 / 2}}{\left(8 n^{3}+18 n+1\right)^{2}} \\
& \times\left[\frac{1}{\left(2 n^{2}-n+8\right)^{2}}+\frac{4 n^{2}+9 n-10}{4(n-1)(n+5)^{2}\left(2 n^{2}-n-10\right)} \cdot\left(\frac{s}{t}\right)^{2}\right] t
\end{aligned}
$$

for $\alpha_{1}(n) \leqq x<1$.

Proof. First, we find a lower bound of $\lambda(x)-\tilde{\lambda}(X(x))$. By means of (1.4), (1.5), (1.5) in [12] and Lemma 7.1 in [13] we have 


$$
\begin{aligned}
& \lambda(x)-\tilde{\lambda}(X(x))=\lambda(x)-\lambda(1)-\{\tilde{\lambda}(X)-\tilde{\lambda}(1)\} \\
& =\int_{x}^{1} \frac{(1-u) d u}{(n-u)^{2}}+\int_{1}^{x} \frac{(u-1)\{n+(n-1) u\} d u}{(n-1) u^{2}(n-u)} \\
& =\int_{0}^{t} \frac{u d u}{(n-1+u)^{2}}+\frac{1}{n-1} \int_{0}^{s} \frac{u\{2 n-1+(n-1) u\} d u}{(1+u)^{2}(n-1-u)} .
\end{aligned}
$$

On the other hand, we see that the function $\frac{2 n-1+(n-1) x}{(1+x)^{2}(n-1-x)}$ is decreasing for $0 \leqq x \leqq \frac{9}{2 n+1}$, because

$$
\frac{d}{d x} \frac{2 n-1+(n-1) x}{(1+x)^{2}(n-1-x)}=-\frac{3 n^{2}-6 n+2+\left(n^{2}-8 n+4\right) x-2(n-1) x^{2}}{(1+x)^{3}(n-1-x)^{2}}<0 .
$$

Hence, we obtain for $0 \leqq x \leqq 9 /(2 n+1)$

$$
\frac{2 n-1+(n-1) x}{(1+x)^{2}(n-1-x)} \geqq\left[\frac{2 n-1+(n-1) x}{(1+x)^{2}(n-1-x)}\right]_{x=9 /(2 n+1)}=\frac{(2 n+1)^{2}\left(4 n^{2}+9 n-10\right)}{4(n+5)^{2}\left(2 n^{2}-n-10\right)} .
$$

Thus, from the above expression of $\lambda(x)-\tilde{\lambda}(x)$ in integral we obtain

$$
\lambda(x)-\tilde{\lambda}(X(x))>\frac{t^{2}}{2\left(n-1+t_{0}\right)^{2}}+\frac{(2 n+1)^{2}\left(4 n^{2}+9 n-10\right) s^{2}}{8(n-1)(n+5)^{2}\left(2 n^{2}-n-10\right)}
$$

and by (7.3)

$$
\lambda(x)-\tilde{\lambda}(X(x))>\frac{(2 n+1)^{2} t^{2}}{2\left(2 n^{2}-n+8\right)^{2}}+\frac{(2 n+1)^{2}\left(4 n^{2}+9 n-10\right) s^{2}}{8(n-1)(n+5)^{2}\left(2 n^{2}-n-10\right)}
$$

$$
\text { for } \alpha_{1}(n) \leqq x<1 \text {. }
$$

Second, we find a lower bound of $F_{2}(x)$. By means of the facts in $\S 3$ in [12], we have for $0<x<1$

$$
\begin{aligned}
F_{2}(x) & =-P_{2}(x) B+(n-x)^{n-1} P_{3}(x)=P_{2}(x)\left\{\frac{(n-x)^{n-1} P_{3}(x)}{P_{2}(x)}-B\right\} \\
& >P_{2}(1) \int_{1}^{x} \frac{d}{d u}\left\{\frac{(n-u)^{n-1} P_{3}(u)}{P_{2}(u)}\right\} d u \\
& =12(n-1)^{2} \int_{1}^{x} \frac{n(n-1)(n-u)^{n-2}(u-1)^{3} Q_{2}(u) d u}{\left(P_{2}(u)\right)^{2}} \\
& =12 n(n-1)^{3} \int_{x}^{1} \frac{(n-u)^{n-2}(1-u)^{3} Q_{2}(u) d u}{\left(P_{2}(u)\right)^{2}} .
\end{aligned}
$$

Since we obtain from (3.2) and (3.6) in [12]

$$
\begin{aligned}
Q_{2}(u) & =(2 n+1) u^{2}-\left(8 n^{2}-2 n+9\right) u+4 n\left(2 n^{2}-2 n+3\right) \\
& >Q_{2}(1)=8(n-1)\left(n^{2}-n+1\right),
\end{aligned}
$$




$$
\begin{array}{r}
P_{2}(u)=(2 n+1) u^{2}-2\left(2 n^{2}+5 n-4\right) u+16 n^{2}-16 n+3 \\
<P_{2}(x)<P_{2}\left(\alpha_{1}\right)<P_{2}\left(\frac{2 n-8}{2 n+1}\right)=\frac{3\left(8 n^{3}+18 n+1\right)}{2 n+1} \\
\text { for } x<u<1, \quad \alpha_{1}(n) \leqq x<1 .
\end{array}
$$

Thus, we obtain

$$
F_{2}(x)>12 n(n-1)^{3} \cdot \frac{(n-1)^{n-2} \cdot 8(n-1)\left(n^{2}-n+1\right) \cdot(2 n+1)^{2}}{9\left(8 n^{3}+18 n+1\right)^{2}} \cdot \int_{x}^{1}(1-u)^{3} d u
$$

i.e.

$$
F_{2}(x)>\frac{8 B n(n-1)^{3}\left(n^{2}-n+1\right)(2 n+1)^{2} t^{4}}{3\left(8 n^{3}+18 n+1\right)^{2}} \quad \text { for } \alpha_{1}(n) \leqq x<1 .
$$

Third, since $x^{2} \sqrt{n-x}$ is increasing for $0<x<(4 / 5) n$, we obtain

$$
\begin{aligned}
x^{2} \sqrt{n-x} & \geqq \alpha_{1}{ }^{2} \sqrt{n-\alpha_{1}}<\left(\frac{2 n-8}{2 n+1}\right)^{2} \sqrt{n-\frac{2 n-8}{2 n+1}} \\
& =\frac{4(n-4)^{2} \sqrt{2 n^{2}-n+8}}{(2 n+1)^{5 / 2}} \quad \text { for } \alpha_{1}(n) \leqq x<1 .
\end{aligned}
$$

Finally, combining these evaluations, we obtain

$$
\begin{aligned}
U_{0}(x) & =\frac{x^{2} \sqrt{n-x}}{(1-x)^{5}} \cdot F_{2}(x) \cdot\{\lambda(x)-\tilde{\lambda}(X(x))\} \\
> & \frac{1}{t^{5}} \frac{4(n-4)^{2} \sqrt{2 n^{2}-n+8}}{(2 n+1)^{5 / 2}} \cdot \frac{8 B n(n-1)^{3}\left(n^{2}-n+1\right)(2 n+1)^{2} t^{4}}{3\left(8 n^{3}+18 n+1\right)^{2}} \\
& \times \frac{(2 n+1)^{2}}{2}\left[\frac{1}{\left(2 n^{2}-n+8\right)^{2}}+\frac{4 n^{2}+9 n-10}{4(n-1)(n+5)^{2}\left(2 n^{2}-n-10\right)}\left(\frac{s}{t}\right)^{2}\right] t^{2} \\
= & \frac{16 B}{3} \cdot \frac{n(n-1)^{3}(n-4)^{2}\left(n^{2}-n+1\right)(2 n+1)^{3 / 2}\left(2 n^{2}-n+8\right)^{1 / 2}}{\left(8 n^{3}+18 n+1\right)^{2}} \\
\quad & \times\left[\frac{1}{\left(2 n^{2}-n+8\right)^{2}}+\frac{4 n^{2}+9 n-10}{4(n-1)(n+5)^{2}\left(2 n^{2}-n-10\right)}\left(\frac{s}{t}\right)^{2}\right] t
\end{aligned}
$$

for $\alpha_{1}(n) \leqq x<1$.

Q.E.D.

LEMMA 7.2. When $n \geqq 10$, we have

$$
\begin{aligned}
U_{4}(x) & >\frac{1}{96} \cdot \frac{n^{2}(n+2)^{n-2}(2 n-5)^{n-2}}{(n-1)^{4}(2 n+1)^{n}} \cdot\left(8 n^{3}-24 n^{2}-18 n+61\right) \\
& \times\left(8 n^{4}-12 n^{3}-28 n^{2}+27 n+5\right) \cdot \frac{(1+s) s}{\sqrt{n-1-s}} \text { for } \alpha_{1}(n) \leqq x<1 .
\end{aligned}
$$


Proof. As in Lemma 7.1, we have

$$
\begin{aligned}
F_{2}(X) & >P_{2}\left(\frac{2 n+10}{2 n+1}\right) \int_{1}^{x} \frac{n(n-1)(n-u)^{n-2}(u-1)^{3} Q_{2}(u) d u}{\left\{P_{2}(u)\right\}^{2}} \\
& >\frac{1}{\left\{P_{1}(1)\right\}^{2}} \cdot P_{2}\left(\frac{2 n+10}{2 n+1}\right) \cdot Q_{2}\left(\frac{2 n+10}{2 n+1}\right) \cdot n(n-1)\left(n-\frac{2 n+10}{2 n+1}\right)^{n-2} \cdot \frac{s^{4}}{4}
\end{aligned}
$$

for $\alpha_{1}(n) \leqq x<1$. Since we have

$$
\begin{aligned}
& P_{2}(1)=12(n-1)^{2}, \\
& P_{2}\left(\frac{2 n+10}{2 n+1}\right)=\frac{3\left(8 n^{3}-24 n^{2}-18 n+61\right)}{2 n+1}, \\
& Q_{2}\left(\frac{2 n+10}{2 n+1}\right)=\frac{2\left(8 n^{4}-12 n^{3}-28 n^{2}+27 n+5\right)}{2 n+1},
\end{aligned}
$$

we obtain

$$
\begin{aligned}
& F_{2}(X)>\frac{1}{12^{2}(n-1)^{4}} \cdot \frac{3\left(8 n^{3}-24 n^{2}-18 n+61\right)}{2 n+1} \\
& \quad \times \frac{2\left(8 n^{4}-12 n^{3}-28 n^{2}+27 n+5\right)}{2 n+1} \cdot \frac{n(n-1)\left(2 n^{2}-n-10\right)^{n-2}}{(2 n+1)^{n-2}} \cdot \frac{s^{4}}{4},
\end{aligned}
$$

i. e.

$$
\begin{aligned}
F_{2}(X) & >\frac{1}{96} \cdot \frac{n(n+2)^{n-2}(2 n-5)^{n-2}}{(n-1)^{3}(2 n+1)^{n}} \cdot\left(8 n^{3}-24 n^{2}-18 n+61\right) \\
& \times\left(8 n^{4}-12 n^{3}-28 n^{2}+27 n+5\right) s^{4} \quad \text { for } 1<X \leqq \frac{2 n+10}{2 n+1} .
\end{aligned}
$$
(7.7).

Thus, we obtain easily from the expressions of $U_{3}(x)$ and $U_{4}(x)$ the inequality

§8. Evaluations of $U_{1}(x), U_{2}(x), U_{5}(x)$ and $U_{6}(x)$ for $\alpha_{1}(n) \leqq x<1$.

LEMMA 8.1. When $n \geqq 4$, we have

$$
U_{1}(x)<\frac{n(n-1)(1-t)^{2}(n-1+t)^{n-5 / 2}}{2(n-1)+t} \cdot\left(2 n-1+\frac{3}{4} t\right) \text { for } 0<x<1 .
$$

Proof. By means of the facts regarding $f_{0}(x)$ in $\S 2$ of [12] and (1.9) we have

$$
\begin{aligned}
U_{1}(x) & =\frac{3 x^{2} f_{0}(x)}{(x-1)^{3} \sqrt{n-x}} \\
& =\frac{3 x^{2}(2 n-1-x)}{(x-1)^{3} \sqrt{n-x}}\left[B-\frac{(n-x)^{n-1}\left\{n-x+(n-1) x^{2}\right\}}{2 n-1-x}\right]
\end{aligned}
$$




$$
\begin{aligned}
& =\frac{3 x^{2}(2 n-1-x)}{(x-1)^{3} \sqrt{n-x}} \int_{x}^{1} \frac{n(n-1)(n-u)^{n-2}(u-1)^{2}(u-2 n) d u}{(2 n-1-u)^{2}} \\
& =\frac{3 n(n-1)(1-t)^{2}(2 n-2+t)}{t^{3} \sqrt{n-1+t}} \int_{0}^{t} \frac{(n-1+u)^{n-2} u^{2}(2 n-1+u) d u}{(2 n-2+u)^{2}} .
\end{aligned}
$$

Since the function $\frac{(n-1+x)^{n-2}}{(2 n-2+x)^{2}}$ is increasing, because

$$
\frac{d}{d x} \frac{(n-1+x)^{n-2}}{(2 n-2+x)^{2}}=\frac{(n-1+x)^{n-3}\{2(n-1)(n-3)+(n-4) x\}}{(2 n-2+x)^{3}}>0
$$

for $x>0$, when $n \geqq 4$, we have

$$
\frac{(n-1+u)^{n-2}}{(2 n-2+u)^{2}}<\frac{(n-1+t)^{n-2}}{(2 n-2+t)^{2}} \quad \text { for } 0<u<t .
$$

Hence, we obtain

$$
\begin{aligned}
& U_{1}(x)<\frac{3 n(n-1)(1-t)^{2}(n-1+t)^{n-5 / 2}}{t^{3}\{2(n-1)+t\}} \int_{0}^{t} u^{2}(2 n-1+u) d u \\
& =\frac{n(n-1)(1-t)^{2}(n-1+t)^{n-5 / 2}}{2(n-1)+t} \cdot\left(2 n-1+\frac{3}{4} t\right) . \quad \text { Q. E. D. }
\end{aligned}
$$

LEMMA 8.2. When $n>3$, we have

$$
U_{2}(x)>\frac{B}{3} \cdot \frac{n^{2}}{(n-1)^{2}} \cdot \frac{(1-t)^{2}}{\sqrt{n-1+t}} \cdot\{3(n-1)+2 t\} \quad \text { for } 0<x<1 .
$$

Proof. By means of (2.8) we have

$$
\begin{aligned}
B-\phi(x) & =\phi(1)-\phi(x)=n \int_{x}^{1}(1-u)(n-u)^{n-2} d u \\
& =n \int_{0}^{t} u(n-1+u)^{n-2} d u>n(n-1)^{n-3} \int_{0}^{t} u(n-1+u) d u \\
& =n(n-1)^{n-3} t^{2}\left(\frac{n-1}{2}+\frac{t}{3}\right)
\end{aligned}
$$

from which we obtain immediately (8.2) by (1.10).

Q.E.D.

LEMMA 8.3. When $n \geqq 4$, we have

$$
U_{5}(x)>\frac{n(n-1)(1+s)^{2}(n-1-s)^{n-5 / 2}}{2(n-1)-s} \cdot\left(2 n-1-\frac{3}{4} s\right)
$$

for $\alpha_{1}(n) \leqq x<1$. 
Proof. By means of an analogous way as to $U_{1}(x)$, we obtain from (1.9) and (1.13)

$$
\begin{aligned}
U_{5}(x) & =\frac{3 X^{2} f_{0}(X)}{(X-1)^{3} \sqrt{n-X}} \\
& =\frac{3 n(n-1)(1+s)^{2}(2 n-2-s)}{s^{3} \sqrt{n-1-s}} \int_{0}^{s} \frac{(n-1-u)^{n-2} u^{2}(2 n-1-u) d u}{(2 n-2-u)^{2}} .
\end{aligned}
$$

Since the function $\frac{(n-1-x)^{n-2}}{(2 n-2-x)^{2}}$ is decreasing for $0<x<1$, when $n \geqq 4$ and $0<$ $s \leqq s_{0}=9 /(2 n+1)$, we obtain

$$
\begin{aligned}
U_{5}(x) & >\frac{3 n(n-1)(1+s)^{2}(2 n-2-s)}{s^{3} \sqrt{n-1-s}} \cdot \frac{(n-1-s)^{n-2}}{(2 n-2-s)^{2}} \int_{0}^{s} u^{2}(2 n-1-u) d u \\
& =\frac{n(n-1)(1+s)^{2}(n-1-s)^{n-5 / 2}}{2(n-1)-s} \cdot\left(2 n-1-\frac{3}{4} s\right) \text { for } \alpha_{1}(n) \leqq x<1 .
\end{aligned}
$$

Q.E.D.

LEMMA 8.4. When $n \geqq 4$, we have

$$
U_{6}(x)<\frac{B}{3} \cdot \frac{n^{2}}{(n-1)^{2}} \cdot \frac{(1+s)^{2}}{\sqrt{n-1-s}}\{3(n-1)-2 s\} \quad \text { for } \alpha_{1}(n) \leqq x<1 .
$$

Proof. By means of an analogous way as to $U_{2}(x)$, we obtain from (1.10) and (1.13)

$$
\begin{aligned}
U_{6}(x) & =\frac{2 n X^{2}\{B-\psi(X)\}}{(X-1)^{2} \sqrt{n-X}}=\frac{2 n^{2} X^{2}}{(X-1)^{2} \sqrt{n-X}} \int_{0}^{s} u(n-1-u)^{n-2} d u \\
& <\frac{2 n^{2}(n-1)^{n-3} X^{2}}{(X-1)^{2} \sqrt{n-X}} \int_{0}^{s} u(n-1-u) d u \\
& =\frac{B}{3} \cdot \frac{n^{2}}{(n-1)^{2}} \cdot \frac{(1+s)^{2}}{\sqrt{n-1-s}}\{3(n-1)-2 s\},
\end{aligned}
$$

if $1<X \leqq n-1$ and $n>3$. Then condition : $(2 n+10) /(2 n+1) \leqq n-1$ is equivalent to $2 n^{2}-3 n-11 \geqq 0$, which is satisfied for $n \geqq 4$. Hence, we obtain (8.4), when $n \geqq 4$.

Q.E.D.

§. Evaluation of $V(x, X(x))$ for $\alpha_{1}(n) \leqq x<1$.

We shall evaluate $V(x, X(x))$ for $\alpha_{1}(n) \leqq x<1$ and show that it is positive, when $n \geqq 84$, which implies the result described in Introduction.

PROPOSITION 4. The function $\left(X_{n}(x)-1\right) /(1-x)$ is monotone decreasing with respect to $x$ for $0<x<1$, when $n>2$.

Proof. Since we have 
188

$$
\begin{aligned}
\frac{d}{d x}\left(\frac{X_{n}(x)-1}{1-x}\right) & =\frac{1}{(1-x)^{2}}\left\{(1-x) X_{n}^{\prime}(x)+X_{n}(x)-1\right\} \\
& =-\frac{X-1}{x(n-x)}\left\{\frac{X(n-X)}{(X-1)^{2}}-\frac{x(n-x)}{(1-x)^{2}}\right\}, \quad X=X_{n}(x),
\end{aligned}
$$

by $d X / d x=\{(1-x) / x(n-x)\} \cdot\{X(n-X) /(1-X)\}$, it is sufficient to prove

$$
\frac{X(n-X)}{(X-1)^{2}}>\frac{x(n-x)}{(1-x)^{2}} \quad \text { for } 0<x<1
$$

which is equivalent to

$$
(X-1)^{2}(n-X)^{n-2}<(1-x)^{2}(n-x)^{n-2} \quad \text { for } 0<x<1 .
$$

On the other hand, we have

$$
\frac{d}{d x}(1-x)^{2}(n-x)^{n-2}=-(1-x)(n-x)^{n-3}(3 n-2-n x) .
$$

Since we have

and

$$
\begin{aligned}
(1-x)^{2}(n-x)^{n-2} & =4\left(\frac{n-1}{n}\right)^{n}(n-2)^{n-2} & & \text { at } x=\frac{3 n-2}{n}, \\
& =n^{n-2} & & \text { at } x=0
\end{aligned}
$$

$$
n^{n-2}>4\left(\frac{n-1}{n}\right)^{n}(n-2)^{n-2} \quad \text { for } n>2
$$

because $((n-1) / n)^{n}((n-2) / n)^{n-2}$ is decreasing with respect to $n$ for $n>2$ as is easily proved and $\lim _{n \rightarrow 2}((n-1) / n)^{n}((n-2) / n)^{n-2}=1 / 4$, there exists a real number $\xi$ $(0<\xi<1)$ uniquely determined by

$$
(1-\xi)^{2}(n-\xi)^{n-2}=4\left(\frac{n-1}{n}\right)^{n}(n-2)^{n-2} .
$$

Then, (9.2) holds clearly for $0<x<\xi$ and $x<1$ sufficiently near 1 by means of (8.12) in [13]. Now, we suppose that (9.2) does not hold at some point of $x$, then there exist a value $y(\xi \leqq y<1)$ such that

$$
(Y-1)^{2}(n-Y)^{n-2}=(1-y)^{2}(n-y)^{n-2}, \quad Y=X_{n}(y)
$$

and

$$
\left[\frac{d}{d x}\left\{(X-1)^{2}(n-X)^{n-2}-(1-x)^{2}(n-x)^{n-2}\right\}\right]_{x=y} \leqq 0,
$$

i. e. 


$$
\begin{gathered}
{\left[-(1-X)(n-X)^{n-3}(3 n-2-n X) \frac{1-x}{x(n-x)} \cdot \frac{X(n-X)}{1-X}\right.} \\
\left.+(1-x)(n-x)^{n-3}(3 n-2-n x)\right]_{x=y} \\
=\frac{1-y}{y(n-y)}\left[-Y(n-Y)^{n-2}(3 n-2-n Y)+y(n-y)^{n-2}(3 n-2-n y)\right] \leqq 0,
\end{gathered}
$$

i.e.

$$
Y(3 n-2-n Y)(n-Y)^{n-2} \geqq y(3 n-2-n y)(n-y)^{n-2} .
$$

Adding (9.3) multiplied with $n-1$, (9.4) and $-Y(n-Y)^{n-1}=-y(n-y)^{n-1}$ in the both sides respectively, we obtain

$$
(n-1)(n-Y)^{n-2} \geqq(n-1)(n-y)^{n-2} .
$$

This is a contradiction, because $0<y<1<Y<n, n>2$. Thus we obtain this lemma.

Q.E.D.

LEMMA 9.1. When $n \geqq 10$, we have

$$
\begin{aligned}
& \frac{V(x, X(x))}{s B \sqrt{n-1}}>\frac{112}{27} \cdot \frac{n(n-1)^{5 / 2}(n-4)^{2}\left(n^{2}-n+1\right)(2 n+1)^{3 / 2}}{\left(8 n^{3}+18 n+1\right)^{2}} \\
& \left.\frac{\left(2 n^{2}-n+8\right)^{1 / 2}}{\left(2 n^{2}-n+8\right)^{2}}+\frac{1}{4(n-1)(n+5)^{2}\left(2 n^{2}-n-10\right)}\right]
\end{aligned}
$$

$$
\begin{aligned}
& +\frac{1}{96} \cdot \frac{n^{2}(n+2)^{n-2}(2 n-5)^{n-2}}{(n-1)^{n+4}(2 n+1)^{n}} \cdot\left(8 n^{3}-24 n^{2}-18 n+61\right)\left(8 n^{4}-12 n^{3}-28 n^{2}+27 n+5\right) \\
& -\frac{s+t}{s} \cdot \frac{n}{12(n-1)^{n-3 / 2}} \cdot \frac{1}{(2 n+1)^{n-3 / 2}(n+2)^{3 / 2}(2 n-5)^{3 / 2}\left(4 n^{2}-2 n+7\right)^{2}} \\
& \times\left[4 n(n-1)^{n-4}(n+5)(2 n+1)^{n-3}\left(4 n^{2}-2 n+7\right)^{2}\left(48 n^{4}-52 n^{3}+342 n^{2}-198 n+616\right)\right. \\
& -3(n-4)(n+2)^{n-2}(2 n-5)^{n-2}\left(256 n^{6}-992 n^{5}+80 n^{4}+4120 n^{3}\right. \\
& \left.\left.-3592 n^{2}-5474 n+3010\right)\right] \quad \text { for } \alpha_{1}(n) \leqq x<1 .
\end{aligned}
$$

Proof. By virtue of Lemmas 7.1, 7.2, 8.1, 8.2, 8.3 and 8.4, we obtain an evaluation of $V(x, X(x))$ as follows: When $n \geqq 10$, for $\alpha_{1}(n) \leqq x<1$, we have

$$
\begin{aligned}
& V(x, X(x))=U_{0}(x)-U_{1}(x)+U_{2}(x)+U_{4}(x)+U_{5}(x)-U_{6}(x) \\
& >\frac{16 B}{3} \cdot \frac{n(n-1)^{3}(n-4)^{2}\left(n^{2}-n+1\right)(2 n+1)^{3 / 2}\left(2 n^{2}-n+8\right)^{1 / 2}}{\left(8 n^{3}+18 n+1\right)^{2}} \\
& \times\left[\frac{1}{\left(2 n^{2}-n+8\right)^{2}}+\frac{4 n^{2}+9 n-10}{4(n-1)(n+5)^{2}\left(2 n^{2}-n-10\right)}\left(\frac{s}{t}\right)^{2}\right] t
\end{aligned}
$$


(9.6)

$$
\begin{aligned}
& +\frac{1}{96} \cdot \frac{n^{2}(n+2)^{n-2}(2 n-5)^{n-2}}{(n-1)^{4}(2 n+1)^{n}} \cdot\left(8 n^{3}-24 n^{2}-18 n+61\right)\left(8 n^{4}-12 n^{3}\right. \\
& \left.-28 n^{2}+27 n+5\right) \cdot \frac{(1+s) s}{\sqrt{n-1-s}} \\
& +n(n-1)\left[\frac{(1+s)^{2}(n-1-s)^{n-5 / 2}}{2(n-1)-s} \cdot\left(2 n-1-\frac{3}{4}\right)\right. \\
& \left.-\frac{(1-t)^{2}(n-1+t)^{n-5 / 2}}{2(n-1)+t}\left(2 n-1+\frac{3}{4} t\right)\right] \\
& -\frac{B}{3} \cdot \frac{n^{2}}{(n-1)^{2}}\left[\frac{(1+s)^{2}}{\sqrt{n-1-s}} \cdot\{3(n-1)-2 s\}-\frac{(1-t)^{2}}{\sqrt{n-1+t}} \cdot\{3(n-1)+2 t\}\right] .
\end{aligned}
$$

When $n \geqq 10$, we obtain from Lemma 6.2, Lemma 6.3 and Proposition 4

$$
1<\frac{s}{t}<\frac{s_{0}}{t_{0}}<\frac{9}{7} \quad \text { for } 0<t<t_{0} .
$$

Regarding the right hand side of (9.6), we have the following facts. First, we have

$$
\frac{1+s}{\sqrt{n-1-s}}>\frac{1}{\sqrt{n-1}} \quad \text { for } 0<s<s_{0}
$$

Second, we have

$$
\frac{d}{d x} \frac{(1+x)^{2}(n-1-x)^{n-5 / 2}\left(2 n-1-\frac{3}{4} x\right)}{2(n-1)-x}=\frac{(1+x)(n-1-x)^{n-\pi / 2} R_{3}(x)}{8\{2(n-1)-x\}^{2}},
$$

where

$$
\begin{aligned}
R_{3}(x):= & 4(n-1)\left(8 n^{2}+n-1\right)-\left(32 n^{3}-40 n^{2}+38 n-10\right) x \\
& +\left(28 n^{2}-32 n+9\right) x^{2}-3(2 n-1) x^{3} .
\end{aligned}
$$

Furthermore, we have for $x \leqq 1$

$$
\begin{aligned}
R_{3}^{\prime}(x) & =-\left(32 n^{3}-40 n^{2}+38 n-10\right)+2\left(28 n^{2}-32 n+9\right) x-9(2 n-1) x^{2} \\
& <-32 n^{3}+40 n^{2}-38 n+10+2\left(28 n^{2}-32 n+9\right) \\
& =-2\left(16 n^{3}-48 n^{2}+51 n-14\right)<0,
\end{aligned}
$$

when $n>2$, and hence

$$
R_{3}(x) \geqq R_{3}(1)=2\left(20 n^{2}-42 n+13\right)>0 .
$$

Thus, we see that $\frac{(1+x)^{2}(n-1-x)^{n-5 / 2}\left(2 n-1-\frac{3}{4} x\right)}{2(n-1)-x}$ is increasing for $-1<x<1$.

Using the mean value theorem and (9.9), we obtain 


$$
\begin{aligned}
& \frac{(1+s)^{2}(n-1-s)^{n-5 / 2}}{2(n-1)-s} \cdot\left(2 n-1-\frac{3}{4} s\right)-\frac{(1-t)^{2}(n-1+t)^{n-5 / 2}}{2(n-1)+t} \cdot\left(2 n-1+\frac{3}{4} t\right) \\
& >(s+t) \cdot \frac{(1-t)(n-1-s)^{n-7 / 2}}{8\{2(n-1)+t\}^{2}} \cdot R_{3}(s) \\
& >(s+t) \cdot \frac{\left(1-t_{0}\right)\left(n-1-s_{0}\right)^{n-7 / 2}}{8\left\{2(n-1)+t_{0}\right\}^{2}} \cdot R_{3}\left(s_{0}\right) \\
& >(s+t) \cdot \frac{\left(1-\frac{9}{2 n+1}\right)\left(n-1-\frac{9}{2 n+1}\right)^{n-7 / 2}}{8\left\{2(n-1)+\frac{9}{2 n+1}\right\}^{2}} \cdot R_{3}\left(\frac{9}{2 n+1}\right) \\
& \quad=(s+t) \cdot \frac{(n-4)\left(2 n^{2}-n-10\right)^{n-7 / 2}}{4\left(4 n^{2}-2 n+7\right)^{2}(2 n+1)^{n-3 / 2}} \cdot\left(256 n^{6}-992 n^{5}+80 n^{4}\right. \\
& \left.\quad+4120 n^{3}-3592 n-5474 n+3010\right) .
\end{aligned}
$$

Third, we have

$$
\frac{d}{d x} \frac{(1+x)^{2}\{3(n-1)-2 x\}}{\sqrt{n-1-x}}=-\frac{(1+x) \mathrm{R}_{2}(x)}{2(n-1-x)^{3 / 2}},
$$

where

$$
R_{2}(x):=(n-1)(12 n-13)-(21 n-23) x+10 x^{2} .
$$

Since $(21 n-23)^{2}-40(n-1)(12 n-13)=-\left(39 n^{2}-34 n-9\right)<0$ for $n>2$, we have $R_{2}(x)>0$. Thus, we see that $\frac{(1+x)^{2}\{3(n-1)-2 x\}}{\sqrt{n-1-x}}$ is increasing for $-1<x<1$. Using the mean value theorem and (9.12), we obtain

$$
\begin{aligned}
& \frac{(1+s)^{2}\{3(n-1)-2 s\}}{\sqrt{n-1-s}}-\frac{(1-t)^{2}\{3(n-1)+2 t\}}{\sqrt{n-1+t}} \\
& <(s+t) \cdot \frac{1+s}{2(n-1-s)^{3 / 2}} \cdot R_{2}(-t)<(s+t) \cdot \frac{(1+s) R_{2}(-s)}{2(n-1-s)^{3 / 2}} \\
& <(s+t) \cdot \frac{\left(1+\frac{9}{2 n+1}\right) R_{2}\left(-\frac{9}{2 n+1}\right)}{2\left(n-1-\frac{9}{2 n+1}\right)^{3 / 2}} \\
& =(s+t) \cdot \frac{(n+5)\left(48 n^{4}-52 n^{3}+342 n^{2}-198 n+616\right)}{\left(2 n^{2}-n-10\right)^{3 / 2}(2 n+1)^{3 / 2}} .
\end{aligned}
$$

Now, from (9.11) and (9.14) we obtain 


$$
\begin{aligned}
& n(n-1)\left[\frac{(1+s)^{2}(n-1-s)^{n-5 / 2}}{2(n-1)-s} \cdot\left(2 n-1-\frac{3}{4} s\right)\right. \\
& \left.\quad-\frac{(1-t)^{2}(n-1+t)^{n-5 / 2}}{2(n-1)+t} \cdot\left(2 n-1+\frac{3}{4} t\right)\right] \\
& \quad-\frac{B}{3} \cdot \frac{n^{2}}{(n-1)^{2}}\left[\frac{(1+s)^{2}\{3(n-1)-2 s\}}{\sqrt{n-1-s}}-\frac{(1-t)^{2}\{3(n-1)+2 t\}}{\sqrt{n-1+t}}\right] \\
& >(s+t)\left[\frac { n ( n - 1 ) ( n - 4 ) ( 2 n ^ { 2 } - n - 1 0 ) ^ { n - 7 / 2 } } { 4 ( 4 n ^ { 2 } - 2 n + 7 ) ^ { 2 } ( 2 n + 1 ) ^ { n - 3 / 2 } } \cdot \left(256 n^{6}-992 n^{5}+80 n^{4}\right.\right. \\
& \left.\quad+4120 n^{3}-3592 n^{2}-5474 n+3010\right) \\
& \left.\quad-\frac{n^{2}(n-1)^{n-3}(n+5)\left(48 n^{4}-52 n^{3}+342 n^{2}-198 n+616\right)}{3\left(2 n^{2}-n-10\right)^{3 / 2}(2 n+1)^{3 / 2}}\right] \\
& =-(s+t) B \sqrt{n-1} \cdot \frac{n}{12(n-1)^{n-3 / 2}} \cdot \frac{1}{(2 n+1)^{n-3 / 2}\left(2 n^{2}-n-10\right)^{3 / 2}} \\
& \quad \frac{\left(4 n^{2}-2 n+7\right)^{2}}{} \cdot\left[4 n(n-1)^{n-4}(n+5)(2 n+1)^{n-3}\left(4 n^{2}-2 n+7\right)^{2}\right. \\
& \times\left(48 n^{4}-52 n^{3}+342 n^{2}-198 n+616\right)-3(n-4)\left(2 n^{2}-n-10\right)^{n-2}\left(256 n^{6}-992 n^{5}\right. \\
& \left.\left.+80 n^{4}+4120 n^{3}-3592 n^{2}-5474 n+3010\right)\right] .
\end{aligned}
$$
(9.5).

Thus, combining (9.7), (9.8) and (9.15) with (9.6), we obtain the inequality

$\S 10$. A proof of positiveness of $V(x, X(x))$ for $\alpha_{1}(n) \leqq x<1$.

First of all, we notice that the first two terms and the third term of the right hand side of (9.5) are of order 1 and 0 with respect to $n$, respectively.

LEMMA 10.1. When $n \geqq 10$, we have

$$
\frac{112}{27} \cdot \frac{(n-1)^{5 / 2}(n-4)^{2}\left(n^{2}-n+1\right)(2 n+1)^{3 / 2}\left(2 n^{2}-n+8\right)^{1 / 2}}{\left(8 n^{3}+18 n+1\right)^{2}}
$$

$$
\times\left[\frac{1}{\left(2 n^{2}-n+8\right)^{2}}+\frac{4 n^{2}+9 n-10}{4(n-1)(n+5)^{2}\left(2 n^{2}-n-10\right)}\right]>\frac{2}{3} \cdot\left(\frac{4}{9}\right)^{4} \cdot \frac{7 \cdot 91 \cdot \sqrt{110}}{101 \cdot 101} .
$$

Proof. Supposing $n \geqq 10$ and setting $1 / n=u$, we have (the left hand side of (10.1))

$$
=\frac{4^{2} \cdot 7}{3^{3}} \cdot \frac{(1-u)^{5 / 2}(1-4 u)^{2}\left(1-u+u^{2}\right)(2+u)^{3 / 2}\left(2-u+8 u^{2}\right)^{1 / 2}}{\left(8+18 u^{2}+u^{3}\right)^{2}}
$$




$$
\begin{aligned}
& \times\left[\frac{u}{\left(2-u+8 u^{2}\right)^{2}}+\frac{4+9 u-10 u^{2}}{4(1-u)(1+5 u)^{2}\left(2-u-10 u^{2}\right)}\right] \\
> & \frac{4^{2} \cdot 7}{3^{3}} \cdot \frac{\left(\frac{9}{10}\right)^{5 / 2} \cdot\left(\frac{3}{5}\right)^{2} \cdot \frac{91}{100} \cdot 2^{3 / 2} \cdot \frac{1}{10}(198)^{1 / 2}}{\frac{(8181)^{2}}{10^{6}}} \cdot\left[0+\frac{4}{4 \cdot \frac{9^{3}}{2 \cdot 10^{2}}}\right] \\
= & \frac{2}{3} \cdot\left(\frac{4}{9}\right)^{4} \cdot \frac{7 \cdot 91 \cdot \sqrt{110}}{101 \cdot 101} .
\end{aligned}
$$

In this computation, we have used the fact the function $(1-u)(1+5 u)^{2}\left(2-u-10 u^{2}\right)$ is increasing for $0<u \leqq 1 / 10$. In fact, we have

$$
\begin{aligned}
& \frac{d}{d u}(1-u)(1+5 u)^{2}\left(2-u-10 u^{2}\right) \\
= & (1+5 u)\left\{3(3-5 u)\left(2-u-10 u^{2}\right)-(1-u)(1+5 u)(1+20 u)\right\} \\
> & (1+5 u)\left\{3 \cdot \frac{5}{2} \cdot\left(2-\frac{1}{10}-\frac{1}{10}\right)-1 \cdot\left(1+\frac{1}{2}\right)(1+2)\right\}=9(1+5 u)>0
\end{aligned}
$$

for $0<u \leqq 1 / 10$ and so

$$
(1-u)(1+5 u)^{2}\left(2-u-10 u^{2}\right) \leqq \frac{9}{10} \cdot \frac{9}{4} \cdot \frac{9}{5}=\frac{9^{3}}{2 \cdot 10^{2}} \quad \text { for } 0<u \leqq \frac{1}{10} .
$$

LEMMA 10.2 . When $n \geqq 10$, we have

Q.E.D.

$$
\frac{1}{96} \cdot \frac{n(n+2)^{n-2}(2 n-5)^{n-2}}{(n-1)^{n+4}(2 n+1)^{n}} \cdot\left(8 n^{3}-24 n^{2}-18 n+61\right)\left(8 n^{4}-12 n^{3}-28 n^{2}+27 n+5\right)
$$

$$
>\frac{3^{4} \cdot 7 \cdot 203 \cdot 291 \cdot e_{9} \cdot\left(e_{5}\right)^{2}}{4^{4} \cdot \sqrt{2} \cdot 10^{6} \cdot e^{3}} .
$$

Proof. Supposing $n \geqq 10$ and setting $1 / n=u$, the left hand side of (10.2) is equal to

$$
\frac{1}{2 \cdot 3 \cdot 4^{2}} \cdot \frac{(1+2 u)^{n-2}(2-5 u)^{n-2}}{(1-u)^{n+4}(2+u)^{n}} \cdot\left(8-24 u-18 u^{2}+61 u^{3}\right)\left(8-12 u-28 u^{2}+27 u^{3}+5 u^{4}\right) .
$$

Using the notation

$$
e(n)=e_{n}:=\left(1+\frac{1}{n}\right)^{n} \text { for } n>0 \text {, }
$$

we have

$$
\begin{aligned}
& (1-u)^{n+4}=(1-u)^{5} / e(n-1), \quad(2+u)^{n}=2^{n} \cdot \sqrt{e(2 n)}, \\
& (1+2 u)^{n-2}=\frac{1}{(1+2 u)^{2}}\left(e\left(\frac{n}{2}\right)\right)^{2}, \quad(2-5 u)^{n-2}=2^{n-5 / 2} \sqrt{2-5 u} /\left(e\left(\frac{2 n-5}{5}\right)\right)^{5 / 2} .
\end{aligned}
$$


Hence, putting these expressions into the above one, we obtain

$$
\begin{aligned}
& \frac{1}{2 \cdot 3 \cdot 4^{3}} \sqrt{2} \frac{\sqrt{ } 2-5 u}{(1-u)^{5}(1+2 u)^{2}} \cdot \frac{e(n-1) \cdot\left(e\left(\frac{n}{2}\right)\right)^{2}}{\sqrt{e(2 n)} \cdot\left(e\left(\frac{2 n-5}{5}\right)\right)^{5 / 2}} \\
& \quad \times\left(8-24 u-18 u^{2}+61 u^{3}\right)\left(8-12 u-28 u^{2}+27 u^{3}+5 u^{4}\right) .
\end{aligned}
$$

Then, since we have

$$
-\frac{d}{d u} \frac{\sqrt{2-5 u}}{(1-u)^{5}(1+2 u)^{2}}=-\frac{1-41 u+130 u^{2}}{2(1-u)^{6}(1+2 u)^{3} \sqrt{2-5 u}},
$$

the function $\frac{\sqrt{2}-5 \bar{u}}{(1-u)^{5}(1+2 u)^{2}}$ takes its minimum in the interval $0<u \leqq \frac{1}{10}$ at $u=$ $\frac{41-\sqrt{1161}}{2 \cdot 130}=0.0266405$, because $\frac{41+\sqrt{1161}}{2 \cdot 130}>\frac{1}{10}$, and its minimum is computed as follows: For $u=0.0266405, \sqrt{ } 2-5 u \risingdotseq 1.3663079,(1-u)^{5} \risingdotseq 0.8737080,(1+2 u)^{2} \doteq$ 1.1094008 , and

$$
\frac{\sqrt{ } 2-5 u}{(1-u)^{5}(1+2 u)^{2}} \div \frac{1.3663079}{0.9692923} \doteqdot 1.40959
$$

Therefore, we obtain

$$
\frac{\sqrt{ } 2-5 u}{(1-u)^{5}(1+2 u)^{2}}>\frac{7}{5} \quad \text { for } 0<u \leqq \frac{1}{10} .
$$

Next, the functions $8-24 u-18 u^{2}+61 u^{3}$ and $8-12 u-28 u^{2}+27 u^{3}+5 u^{4}$ are decreasing for $0<u \leqq 1 / 10$ are easily seen, and so we obtain

$$
8-24 u-18 u^{2}+61 u^{3} \geqq 8-\frac{24}{10}-\frac{18}{10^{2}}+\frac{16}{10^{3}}=\frac{3^{3} \cdot 203}{10^{3}},
$$

$$
\begin{gathered}
8-12 u-28 u^{2}+27 u^{3}+5 u^{4} \geqq 8-\frac{12}{10}-\frac{28}{10^{2}}+\frac{27}{10^{3}}+\frac{5}{10^{4}}=\frac{3^{2} \cdot 5^{2} \cdot 291}{10^{4}} \\
\text { for } 0<u \leqq \frac{1}{10} .
\end{gathered}
$$

Thus, combining (10.4) and (10.5) with (10.3) and using the inequality

$$
\frac{e(n-1)\left(e\left(\frac{n}{2}\right)\right)^{2}}{\sqrt{e(2 n)}\left(\mathrm{e}\left(\frac{2 n-5}{5}-\right)\right)^{5 / 2}}>\frac{e_{9} \cdot\left(e_{5}\right)^{2}}{e^{3}} \quad \text { for } n \geqq 10 \text {, }
$$

we obtain the inequality (10.2).

Q.E.D.

LEMMA 10.3. When $n \geqq 10$, we have 


$$
\begin{aligned}
& \frac{n}{12(n-1)^{n-3 / 2}} \cdot \frac{1}{(2 n+1)^{n-3 / 2}(n+2)^{3 / 2}(2 n-5)^{3 / 2}\left(4 n^{2}-2 n+7\right)^{2}} \\
& \times\left[4 n(n-1)^{n-4}(n+5)(2 n+1)^{n-3}\left(4 n^{2}-2 n+7\right)^{2}\left(48 n^{4}-52 n^{3}+342 n^{2}-198 n+616\right)\right. \\
& -3(n-4)(n+2)^{n-2}(2 n-5)^{n-2}\left(256 n^{6}-992 n^{5}+80 n^{4}+4120 n^{3}-3592 n^{2}\right. \\
& -5474 n+3010)]<\frac{10 \cdot 4267 \cdot \sqrt{3 \cdot 5 \cdot 7}}{3^{7} \cdot 7^{2}}-\frac{3 \sqrt{3} \cdot 199147}{7 \cdot 8^{3} \cdot 10^{3}} .
\end{aligned}
$$

Proof. Supposing $n \geqq 10$ and setting $1 / n=u$, the left hand side of (10.6) is equal to

$$
\begin{aligned}
& \frac{1}{12(1+2 u)^{3 / 2}(2-5 u)^{3 / 2}\left(4-2 u+7 u^{2}\right)^{2}} \cdot\left[\frac{4(1+5 u)\left(4-2 u+7 u^{2}\right)^{2}}{(1-u)^{5 / 2}(2+u)^{3 / 2}}\right. \\
& \cdot\left(48-52 u+342 u^{2}-198 u^{3}+616 u^{4}\right)-\frac{3(1-4 u)}{\sqrt{ } 1-u \sqrt{ } 2+u} \cdot\left(\frac{n+2}{n-1}\right)^{n-2} \cdot\left(\frac{2 n-5}{2 n+1}\right)^{n-2} \\
& \left.\cdot\left(256-992 u+80 u^{2}+4120 u^{3}-3592 u^{4}-5474 u^{5}+3010 u^{6}\right)\right] .
\end{aligned}
$$

Since we have

$$
\left(\frac{n+2}{n-1}\right)^{n-2}=-\frac{1-u}{1+2 u}\left(e\left(\frac{n-1}{3}\right)\right)^{3}, \quad\left(\frac{2 n-5}{2 n+1}\right)^{n-2}=\sqrt{\frac{2-5 u}{2+u}}\left(e\left(\frac{2 n-5}{6}\right)\right)^{-3},
$$

substituting these into the above expression, we obtain

$$
\begin{aligned}
& \frac{(1+5 u)\left(48-52 u+342 u^{2}-198 u^{3}+616 u^{4}\right)}{3(1+2 u)^{3 / 2}(2-5 u)^{3 / 2}(1-u)^{5 / 2}(2+u)^{3 / 2}} \\
& -\frac{(1-4 u) \sqrt{1-u}}{4(1+2 u)^{5 / 2}(2-5 u)(2+u)} \cdot \frac{1}{\left(4-2 u+7 u^{2}\right)^{2}} \cdot\left(e\left(\frac{n-1}{3}\right) / e\left(\frac{2 n-5}{6}\right)\right)^{3} \\
& \times\left(256-992 u+80 u^{2}+4120 u^{3}-3592 u^{4}-5474 u^{5}+3010 u^{6}\right) .
\end{aligned}
$$

Now, we estimate the terms in the above expression. The function $(1+5 u)\left(48-52 u+342 u^{2}-198 u^{3}+616 u^{4}\right)$ is increasing for $0<u \leqq 1 / 10$, because

$$
\begin{aligned}
& \frac{d}{d u}(1+5 u)\left(48-52 u+342 u^{2}-198 u^{3}+616 u^{4}\right) \\
= & (1+5 u)\left(-52+684 u-594 u^{2}+2464 u^{3}\right)+5\left(48-52 u+342 u^{2}-198 u^{3}+616 u^{4}\right) \\
> & (1+5 u)(-52)+5\left(48-\frac{52 \cdot 13}{171}+\frac{342 \cdot 13 \cdot 13}{171 \cdot 171}-\frac{198}{1000}\right) \\
> & -\frac{3}{2} \cdot 52+5(48-4+1-1)=142>0,
\end{aligned}
$$

and so we obtain 


$$
\begin{aligned}
& (1+5 u)\left(48-52 u+342 u^{2}-198 u^{3}+616 u^{4}\right) \\
& \leqq \frac{3}{2}\left(48-\frac{52}{10}+\frac{342}{10^{2}}-\frac{198}{10^{3}}+\frac{616}{10^{4}}\right)=\frac{2 \cdot 3^{4} \cdot 4267}{10^{4}} \text { for } 0<u \leqq \frac{1}{10} .
\end{aligned}
$$

The function $(1+2 u)^{3}(2-5 u)^{3}(1-u)^{5}(2+u)^{3}$ is decreasing for $0<u \leqq 1 / 10$, because

$$
\begin{aligned}
& \frac{d}{d u}(1+2 u)^{3}(2-5 u)^{3}(1-u)^{5}(2+u)^{3} \\
& =-(1+2 u)^{2}(2-5 u)^{2}(1-u)^{4}(2+u)^{2}\{(7+8 u)(1+2 u)(2-5 u) \\
& \quad+3(1+20 u)(1-u)(2+u)\}<0
\end{aligned}
$$

and so we obtain

$$
\begin{aligned}
(1+2 u)^{3}(2-5 u)^{3}(1-u)^{5}(2+u)^{3} & >\left(1+\frac{1}{5}\right)^{3}\left(2-\frac{1}{2}\right)^{3}\left(\frac{9}{10}\right)^{5}\left(\frac{21}{10}\right)^{3} \\
& =\frac{2^{3} \cdot 3^{19} \cdot 7^{3}}{10^{11}}
\end{aligned}
$$

hence

$$
\begin{gathered}
(1+2 u)^{3 / 2}(2-5 u)^{3 / 2}(1-u)^{5 / 2}(2+u)^{3 / 2} \geqq \frac{2^{2} \cdot 3^{9} \cdot 7}{10^{6}} \cdot \sqrt{3 \cdot 5 \cdot 7} \\
\text { for } 0<u \leqq \frac{1}{10} .
\end{gathered}
$$

The function $256-992 u+80 u^{2}+4120 u^{3}-3592 u^{4}-5474 u^{5}+3010 u^{6}$ is decreasing for $0<u \leqq 1 / 10$, because its derivative is

$$
\begin{aligned}
& -992+160 u+12360 u^{2}-14368 u^{3}-27370 u^{4}+18060 u^{5} \\
& <-992+16+123.6+0.1806<0,
\end{aligned}
$$

and so we obtain

$$
\begin{gathered}
256-992 u+80 u^{2}+4120 u^{3}-3592 u^{4}-5474 u^{5}+3010 u^{6} \\
>256-\frac{992}{10}+\frac{8}{10}+\frac{412}{10^{2}}-\frac{3592}{10^{4}}-\frac{5474}{10^{5}}+\frac{301}{10^{5}}=\frac{3^{4} \cdot 199147}{10^{5}} \\
\text { for } 0<u \leqq \frac{1}{10} .
\end{gathered}
$$

We obtain also easily

$$
\begin{gathered}
(1-4 u) \sqrt{1-u} \geqq\left(1-\frac{2}{5}\right) \sqrt{1-\frac{1}{10}}=\frac{3^{2}}{5 \cdot \sqrt{ } 10} \quad \text { for } 0<u \leqq \frac{1}{10}, \\
\left(e\left(\frac{n-1}{3}\right) / e\left(\frac{2 n-5}{6}\right)\right)^{3}>1 .
\end{gathered}
$$


The function $(1+2 u)^{5 / 2}(2-5 u)(2+u)$ is increasing for $0<u \leqq 1 / 10$, because

$$
\begin{aligned}
& \frac{d}{d u}\left\{(1+2 u)^{5 / 2}(2-5 u)(2+u)\right\} \\
= & (1+2 u)^{3 / 2}\{(1+2 u)(2-5 u)+5(1-7 u)(2+u)\}>0,
\end{aligned}
$$

and so we obtain

$$
\begin{gathered}
(1+2 u)^{5 / 2}(2-5 u)(2+u) \leqq\left(\frac{6}{5}\right)^{5 / 2} \cdot \frac{3}{2} \cdot \frac{21}{10}=\frac{2^{4} \cdot 3^{4} \cdot 7 \cdot \sqrt{3}}{10^{3} \sqrt{10}} \\
\text { for } 0<u \leqq \frac{1}{10} .
\end{gathered}
$$

We obtain also easily

$$
4-2 u+7 u^{2}<4 \quad \text { for } 0<u \leqq \frac{1}{10} .
$$

Finally, combining these inequalities (10.8) (10.14) with (10.7), we obtain \{the expression (10.7) of $u\}$

$$
\begin{aligned}
& <\frac{\frac{2 \cdot 3^{4} \cdot 4267}{10^{4}}}{3 \times \frac{2^{2} \cdot 3^{9} \cdot 7}{10^{6}} \cdot \sqrt{3 \cdot 5 \cdot 7}}-\frac{\frac{3^{2}}{5 \cdot \sqrt{10}} \times 1 \times \frac{3^{4} \cdot 199147}{10^{5}}}{4 \times \frac{2^{4} \cdot 3^{4} \cdot 7 \cdot \sqrt{3}}{10^{3} \sqrt{10}} \times 4^{2}} \\
& =\frac{10 \cdot 4267 \cdot \sqrt{3 \cdot 5 \cdot 7}}{3^{7} \cdot 7^{2}}-\frac{3 \sqrt{3} \cdot 199147}{2^{3} \cdot 4^{3} \cdot 7 \cdot 10^{3}} \quad(>0) \quad \text { for } 0<u \leqq \frac{1}{10} .
\end{aligned}
$$

Thus, we obtain the inequality (10.6). Q.E.D.

Now, we have reached to a step to evaluate the sign of $V(x, X(x))$ for $\alpha_{1}(n) \leqq x<1$ by Lemma 9.1, Lemma 10.1 Lemma 10.3 as follows.

When $n \geqq 10$, for $\alpha_{1}(n) \leqq x<1$ we obtain

$$
\begin{aligned}
\frac{V(x, X(x))}{s B \sqrt{n-1}}> & n\left[\frac{2}{3} \cdot\left(\frac{4}{9}\right)^{4} \cdot \frac{7 \cdot 91 \cdot \sqrt{110}}{101 \cdot 101}+\frac{3^{4} \cdot 7 \cdot 203 \cdot 291 \cdot e_{9} \cdot\left(e_{5}\right)^{2}}{4^{4} \cdot \sqrt{2} \cdot 10^{6} \cdot e^{3}}\right] \\
& -2\left[\frac{10 \cdot 4267 \sqrt{3 \cdot 5 \cdot 7}}{3^{7} \cdot 7^{2}}-\frac{3 \sqrt{3} \cdot 199147}{2^{3} \cdot 4^{3} \cdot 7 \cdot 10^{3}}\right] .
\end{aligned}
$$

In the following, we evaluate the right hand side of (10.15).

$$
\frac{2}{3} \cdot\left(\frac{4}{9}\right)^{4} \cdot \frac{7 \cdot 91 \cdot \sqrt{110}}{101 \cdot 101}=\frac{326144 \sqrt{110}}{19683 \cdot 10201} \doteqdot 0.017036
$$

Since we have

$$
e_{9} \cdot\left(e_{5}\right)^{2}=\left(\frac{10}{9}\right)^{9}\left(\frac{6}{5}\right)^{10}=\frac{2^{20}}{3^{8} \cdot 10}
$$


hence

$$
\frac{3^{4} \cdot 7 \cdot 203 \cdot 291 \cdot e_{9} \cdot\left(e_{5}\right)^{2}}{4^{4} \cdot \sqrt{2} \cdot 10^{6} \cdot e^{3}}=\frac{4^{6} \cdot 7 \cdot 203 \cdot 291}{3^{4} \cdot \sqrt{2} \cdot 10^{7} \cdot e^{3}} \doteqdot 0.073615 \text {. }
$$

Therefore, we obtain

$$
\frac{2}{3} \cdot\left(\frac{4}{9}\right)^{4} \cdot \frac{7 \cdot 91 \cdot \sqrt{1} 10}{101 \cdot 101}+\frac{3^{4} \cdot 7 \cdot 203 \cdot 291 \cdot e_{9} \cdot\left(e_{5}\right)^{2}}{4^{4} \cdot \sqrt{2 \cdot 10^{6} \cdot e^{3}}} \risingdotseq 0.090651 .
$$

Next, we have

$$
\begin{aligned}
& \frac{10 \cdot 4267 \cdot \sqrt{3 \cdot 5 \cdot 7}}{3^{7} \cdot 7^{2}}-\frac{3 \sqrt{3} \cdot 199147}{2^{3} \cdot 4^{3} \cdot 7 \cdot 10^{3}}=\frac{42670 \cdot \sqrt{105}}{107163}-\frac{597441 \cdot \sqrt{3}}{3584000} \\
& \quad \doteqdot 4.080115-0.288727=3.791388
\end{aligned}
$$

By means of (10.16) and (10.17), we obtain the following formula: When $n \geqq 10$,

$$
\begin{array}{r}
V(x, X(x))>\frac{s B \sqrt{n-1}}{100}(9.0651 n-379.1388 \times 2) \\
\text { for } \alpha_{1}(n) \leqq x<1 .
\end{array}
$$

Therefore, if $n>\frac{379.138 \times 2}{9.0651} \doteqdot 83.6480$, the right hand side of (10.18) is positive. Thus, we obtain

Proposition 4. When $n \geqq 84$, we have

$$
V(x, X(x))>0 \quad \text { for } \alpha_{1}(n) \leqq x<1 .
$$

From Proposition 3 and Proposition 4, we obtain the main theorem.

THEOREM C. The period function $T$ as a function of $\tau$ and $n$ is monotone decreasing with respect to $n \geqq 84$ for any fixed $\tau(0<\tau<1)$.

In the next paper [14], we shall prove that Conjecture $C$ is also true for $16 \leqq n \leqq 84$.

\section{REFERENCES}

[1] S.S. Chern, M. do Carmo and S. Kobayashi, Minimal submanifolds of a sphere with second fundamental form of constant length, Functional Analysis and Related Fields, Springer-Verlag, 1970, 60-75.

[2] S. FuRUYA, On periods of periodic solutions of a certain nonlinear differential equation, Japan-United States Seminar on Ordinary Differential and Functional Equations, Lecture Notes in Mathematics, Springer-Verlag, 243 (1971), 320-323.

[3] W.Y. Hsiang and H.B. Lawson, JR., Minimal submanifolds of low cohomogeneity, J. Diff. Geometry, 5 (1970), 1-38. 
[4] T. Otsuki, Minimal hypersurfaces in a Riemannian manifold of constant curvature, Amer. J. Math., 92 (1970), 145-173.

[5] T. OTsukI, On integral inequalities related with a certain nonlinear differentıal equation, Proc. Japan Acad., 48 (1972), 9-12.

[6] T. Otsuki, On a 2-dimensional Riemannian manifold, Differential Geometry, in honor of K. YANo, Kinokuniya, Tokyo, 1972, 401-414.

[7] T. Otsuki, On a family of Riemannıan manifolds defined on an $m$-disk, Math. J. Okayama Univ., 16 (1973), 85-97.

[8] T. OTsuki, On a bound for periods of solutions of a certain nonlinear differential equation (I), J. Math. Soc. Japan, 26 (1974), 206-233.

[9] T. OTsuki, On a bound for periods of solutions of a certain nonlinear differential equation (II), Funkcıalaj Ekvacioj, 17 (1974), 193-205.

[10] T. Otsuki, Geodesics of $O_{\tilde{n}}^{2}$ and an analysis on a related Riemann surface, Tôhoku Math. J., 28 (1976), 411-427.

[11] T. OTsuki, A certain property of geodesics of the family of Riemannian manifolds $O_{n}^{2}$ (I), Proc. of Japan-United States Seminar on Minimal Submanifolds, including Geodesıcs, Kaiga1, Tokyo, 1978, 173-192.

[12] T. Отsuki, A certain property of geodesics of the family of Riemannian manifolds $O_{n}^{2}$ (II), Kodai Math. J., 2 (1979), 211-242.

[13] T. Otsuki, A certain property of geodesics of the family of Riemannian mantfolds $O_{n}^{2}$ (III), Koda1 Math. J., 4 (1981), 28-70.

[14] T. OTsuki, A certain property of geodesics of the family of Riemannan manifolds $O_{n}^{2}(V)$, to appear in koda1 Math. J.

[15] M. MAeda, AND T. Otsuki, Models of the Riemannian manifolds $O_{\bar{n}}^{2}$ in the Lorentzian 4-space, J. Diff. Geometry, 9 (1974), 97-108.

[16] M. URABE, Computations of periods of a certain nonlinear autonomous oscilations, Study of algorithms of numerical computations, Sûrikaıseki Kenkyûsho Kôkyûroku, 149 (1972), 111-129 (Japanese).

Department of Mathematics

Science University of TOKyo

WaKamiYa-cho 26, SinNJUKU-KU

TOKYO, JAPAN 162 
